\title{
Mit einer Realexperimentreihe Impulse für soziale Innovationen setzen - Realexperimente initiieren, begleiten und beforschen
}

\author{
Helena Trenks, Colette Waitz, Sarah Meyer-Soylu \& Oliver Parodi
}

\section{Kontext}

Das hier beschriebene Vorgehen eignet sich gut für Gruppen von Wissenschaftler(inne)n, die gemeinsam mit zivilgesellschaftlichen Akteuren neue Impulse in ihrem Lebensumfeld anstoßen und gleichzeitig für eine nachhaltige Entwicklung bzw. zukunftsrelevante gesellschaftliche Themen sensibilisieren wollen.

Das Vorgehen zielt darauf ab, mehrere zivilgesellschaftliche Kleingruppen für die Durchführung einzelner Projekte mit experimentellem Charakter (Realexperimente) zu gewinnen und sie bei Aufbau und Durchführung forschend zu begleiten. Diese Projekte sollen dabei immer einen Mehrwert für die Gemeinschaft mit sich bringen und für weitere Interessierte offen und partizipativ angelegt sein.

In der folgenden Beschreibung werden Empfehlungen für den Kooperationsaufbau mit Partnerinstitutionen gegeben. Zudem wird gezeigt, wie sich über einen Wettbewerb interessierte Bürger(innen) - hier verstanden als die Bevölkerung vor Ort (zu den Herausforderungen der Begrifflichkeit s. a. Defila und Di Giulio 2018 sowie Arnold und Piontek 2018) - für die Realexperimente gewinnen lassen. Was bei der Durchführung und Betreuung solcher Experimente aus wissenschaftlicher Sicht zu beachten ist und welche wissenschaftlichen Methoden sich zu ihrer Beforschung eignen, wird im vorliegenden Text ebenfalls dargestellt. Die Reflexion geht abschließend auf die Wahrnehmung und Resonanz der vorgestellten Methoden ein.

\subsection{Reallaborumgebung}

Das Projekt „Quartier Zukunft - Labor Stadt“ (Quartier Zukunft) besteht seit 2012 und hat zum Ziel, ein Stadtquartier - die Karlsruher Oststadt - in ein nachhaltigeres Quartier zu transformieren. Dies soll im engen Schulterschluss von 
Wissenschaft, Bürger(inne)n und weiteren Akteuren (z. B. NGOs, Vereinen, Kirchen, aber auch Gewerbetreibenden und Unternehmen) geschehen (Albiez et al. 2016; Parodi 2016). In einer engen Verbindung von Wissenschaft - des Karlsruher Instituts für Technologie (KIT) - und insbesondere der Bürgerschaft soll ein gemeinsamer Prozess hin zu einer nachhaltigeren Lebensweise angestoßen werden. Seit 2015 wird das Quartier Zukunft ergänzt durch das „Reallabor 131: KIT findet Stadt" (R131), welches den Fokus, ebenfalls mit der Zielsetzung einer Nachhaltigen Entwicklung der Oststadt, auf die Bereiche Energie, Mobilität, Konsum, Soziales und Raum richtet. ${ }^{1}$

Der für das Reallabor Karlsruhe gewählte ,Raum‘ steht hierbei exemplarisch für die Stadtquartiere mitteleuropäischer Städte, die sich in ihren baulichen wie sozialen Beständen den Herausforderungen einer Nachhaltigen Entwicklung im 21. Jahrhundert stellen (müssen). Die Karlsruher Oststadt ist ein heterogenes, gewachsenes Stadtviertel in Zentrumsnähe, in dem rund 22000 Menschen wohnen. Der Anteil der Einpersonenhaushalte ist mit zwei Drittel vergleichsweise hoch, und es gibt einen relativ hohen Anteil an Personen ausländischer Nationalität $(28 \%){ }^{2}$

Das Reallabor Karlsruhe versteht sich nicht nur als Forschungsprojekt im Bereich Nachhaltigkeit, sondern explizit auch als transformativ angelegte Plattform für eine Vielfalt an lokalen Nachhaltigkeitsaktivitäten. Die Absicht ist, die Zivilgesellschaft - individuelle Bürger(innen), Vereine, NGOs, Initiativen etc. - jenseits herkömmlicher Partizipationsoptionen zur aktiven Gestaltung ihres Lebensumfelds zu befähigen (Empowerment-Strategie). Hierfür wurden in den letzten Jahren unterschiedliche Formate gewählt. Etwa eine ganztägige Bürgerversammlung mit ca. 150 Teilnehmer(inne)n zu Beginn der Reallaborarbeit oder ein mehrwöchiges „BürgerForum Nachhaltige Oststadt“ (ca. 300 Beteiligte, als Auftakt des R131). Im Zuge der Etablierung des Reallabors Karlsruhe konnten einige Projekte verstetigt werden, wie beispielsweise Stammtisch, ReparaturCafé, Kleidertauschparty, Pflanzentauschbörse sowie Dialogstände auf der Straße. Die unterschiedlichen Formate und Projekte haben auch zum Ziel, möglichst viele verschiedene gesellschaftliche Gruppen anzusprechen.

Um partizipative Projekte dieser Art erfolgreich durchführen und beforschen zu können, sind eine stabile Reallabor-Infrastruktur vor Ort sowie ein gewisser Bekanntheitsgrad im Reallabor-, Raum' hilfreich, wenn nicht sogar erforderlich (s. dazu auch Beecroft und Parodi 2016; Parodi et al. 2016). Mit der Eröffnung des

1 Im Folgenden werden die beiden transdisziplinären Forschungsprojekte „Quartier Zukunft - Labor Stadt“ und „Reallabor 131: KIT findet Stadt“ (R131) gemeinsam als „Reallabor Karlsruhe“ bezeichnet (s. a. Steckbrief im Anhang zu diesem Buch).

2 Wohl auch dadurch bedingt, dass hier die Landeserstaufnahmeeinrichtung für Flüchtlinge in Baden-Württemberg ihren Sitz hat (Hammer et al. 2014). 
„Zukunftsraums für Nachhaltigkeit und Wissenschaft“, dem Quartiersbüro des Reallabors Karlsruhe in der Oststadt, wurde beides ermöglicht und vorangetrieben, denn damit existiert seit 2015 ein physischer Ort für Nachhaltigkeitsaktivitäten mit Wiedererkennungswert. So hat das Reallabor Karlsruhe seit seinem Start nach und nach einen größeren und weiteren Kreis an aktiven und interessierten Akteuren in der Oststadt aufgebaut. Diese wirken als Teilnehmer(innen), aber auch als Multiplikator(inn)en an den verschiedenen Formaten und Projekten mit bzw. werden (inzwischen) auch selbstständig aktiv (s. Parodi et al. 2016; Seebacher et al. 2018; Waitz und Meyer-Soylu 2016). Entwickelt werden die Aktivitäten des Reallabors dabei nach keinem vordefinierten Fahrplan. Vielmehr dienen die verschiedenen Beteiligungs- und Diskussionsmöglichkeiten mit ihren thematischen Schwerpunkten dazu, explorativ in den Reallabor-, Raum' hineinzuhorchen und aus der Resonanz Projekte zu entwickeln, die dann wiederum mit lokalen Akteuren durchgeführt werden. Dieses ,dialogisch-intuitive Vorgehen' gibt den am Reallabor beteiligten Wissenschaftler(inne)n die Möglichkeit, flexibel lokale Bedürfnisse, sich ändernde Fragestellungen und bereits (im Keim) vorhandene Nachhaltigkeitspotentiale zu adressieren.

\subsection{Die Realexperimentreihe „Dein NachhaltigkeitsExperiment“}

Experimente in Reallaboren werden mit der Intention durchgeführt, in der Lebenswelt der Akteure etwas zu verändern. Sie zeichnen sich durch ein experimentelles Setting aus, in dem Design, Durchführung, Aus- und Verwertung offen sind für zivilgesellschaftliche Beteiligung (Parodi et al. 2016, ausführlicher zu Reallaboren und Realexperimenten s. Arnold und Piontek 2018 sowie Beecroft et al. 2018). Realexperimente sind ergebnisoffen angelegt. Um den thematischen $\mathrm{Zu}-$ schnitt der Realexperimente in Karlsruhe zu verdeutlichen, wurde für diese der Name „Nachhaltigkeitsexperiment“ gewählt.

Ein Realexperiment ist ein Projekt mit offenem Ausgang. Durch diese Prozessoffenheit wird ein größeres Maß an Flexibilität und Aufwand benötigt, als dies in anderen Forschungsvorhaben der Fall ist; Planbarkeit ist nur begrenzt gegeben. Dies wird dadurch verschärft, dass ein Realexperiment in einer nicht zu kontrollierenden, eben realweltlichen Umgebung, stattfindet. Äußere politische Umschwünge, neue lokale Gegebenheiten, aber auch gruppeninterne Prozesse machen sie zu sensiblen und hochdynamischen Unternehmungen.

Im Reallabor Karlsruhe wurde im Sommer 2016 komplementär zu den anderen Reallaboraktivitäten im Quartier eine neunmonatige Reihe von Realexperimenten mit dem Titel „Dein NachhaltigkeitsExperiment“ (DNE) durchgeführt. Der Beitrag ist der Vorgehensweise bei Aufbau und Durchführung einer solchen Realexperimentreihe gewidmet. Die Beschreibung des Vorgehens erfolgt dabei auf zwei Ebenen: Allgemeine Hinweise zur Wahl und Anwendung von Methoden werden 
ergänzt um Erfahrungen aus der Umsetzung von „Dein NachhaltigkeitsExperiment" (DNE), welches zur Illustration dient. Im Reallabor Karlsruhe wurden im Rahmen von DNE transdisziplinäre Realexperimente auf Basis der Themenschwerpunkte „Gemeinschaft“ und „Entschleunigung“ realisiert, welche parallel liefen und von Gruppen von Bürger(inne)n in verschiedenen Bereichen umgesetzt wurden. Die Realexperimentreihe hatte zum Ziel, neben den damals bereits aktiven Bürger(inne)n (vorwiegend aus der gebildeten Mittelschicht, Personen älter als 50 Jahre), insbesondere jüngere Menschen (20-30 Jahre) zu adressieren. Als Startpunkt für DNE wurde gemeinsam mit einem lokalen Kooperationspartner, der Bürgerstiftung Karlsruhe, ein Ideen-Wettbewerb initiiert.

Die im Rahmen von DNE durchgeführten Nachhaltigkeitsexperimente hatten mehrere Zielsetzungen. So sollten die Themen Gemeinschaft und Entschleunigung als Bedingungen Nachhaltiger Entwicklung erforscht, gesellschaftliche Lernprozesse und Wandel angestoßen, Nachhaltigkeit im Stadtraum konkret erfahrbar gemacht und die Sichtbarkeit des Reallabors Karlsruhe erhöht werden. Grundlegend war dabei die Idee, Bürger(innen) zu ermächtigen, selbst tätig zu werden. Die Nachhaltigkeitsexperimente sollten also weitgehend selbstständig durchgeführt werden. Für das Reallabor-Team ${ }^{3}$ als Initiator und wissenschaftlicher Partner lag der Fokus auf der Begleitung der Nachhaltigkeitsexperimente. Der Transformationsprozess bildete die Grundlage, auf der eine integrierende Wissensproduktion aufsetzte. Mit dem thematischen Zuschnitt auf Gemeinschaft und Entschleunigung wurden mit „Dein NachhaltigkeitsExperiment“ keine üblichen Nachhaltigkeitsthemen gewählt, vielmehr wurden diese Themen thesenhaft als Bedingungen der Möglichkeit Nachhaltiger Entwicklung heutiger postmoderner Gesellschaften ins Feld geführt.

Während das Thema Gemeinschaft dialogisch entstand, indem es bei vorhergegangenen Aktivitäten des Reallabors Karlsruhe durch zivilgesellschaftliche Akteure immer wieder eingefordert wurde, entstand das Thema Entschleunigung aus der Beobachtung des Alltags und der Theoriedebatte (vgl. Rosa 2014) und wurde vom Reallabor-Team gesetzt. Neben der Beforschung dieser beiden Themen wurden in DNE auch grundlegende Fragen des Reallabors Karlsruhe adressiert:

- Wie kann Nachhaltigkeit in der Praxis gelingen?

- Welche Rolle kann transdisziplinäre Forschung dabei spielen?

- Wie kann welche Reallaborinfrastruktur dabei helfen?

3 Als „Reallabor-Team“ werden die wissenschaftlichen Mitarbeiter(innen) im Reallabor Karlsruhe bezeichnet. Wird in der allgemeinen Beschreibung der Realexperimentreihe von „Wissenschaftler(innen)“ gesprochen, ist damit das Team der Wissenschaftler(innen) gemeint, das für die Durchführung der Reihe zuständig ist. 
Das Reallabor Karlsruhe will aktiv auf das Lebensumfeld im Quartier einwirken und auch die bei der Entstehung und Umsetzung von Projekten stattfindenden sozialen Prozesse beleuchten. Dafür wurde mit DNE ein besonders intensives Format entwickelt, dessen Teilnehmer(innen) sehr nahe forschend begleitet wurden. Gemeinsam mit dem Kooperationspartner Bürgerstiftung Karlsruhe wurden aus den eingegangenen Projektideen für DNE vier Ideen ausgewählt, die dann von Gruppen von Bürger(inne)n durchgeführt und vom Reallabor-Team begleitet und beforscht wurden. Diese vier Nachhaltigkeitsexperimente im Reallabor Karlsruhe sind im Folgenden kurz beschrieben: Das Experiment „Kreativ-Salon“ schuf einen offenen Raum für Musik, Gesang und Theaterspiel und alles, was Ausdruck von Kreativität sein kann. Im Experiment „Second Future“ wurde ein Label entwickelt, um den Kauf von Secondhand-Produkten als Ausdruck einer unabhängigen, alternativen Lebensweise sichtbar zu machen. Der „Oststadt-Treff für Jung und Alt" war ein Experiment der Nachbarschafts- und Gemeinschaftsbildung, indem ein Ort der Begegnung für Menschen jeden Alters und jeder Herkunft entstand. „Beete \& Bienen“ hatte zum Ziel, Naschbeete für Menschen und Bienen in der Stadt zu fördern und Bienen (zurück) in die Stadt zu holen. ${ }^{4}$

\section{Eine Realexperimentreihe aufbauen und durchführen}

Im Folgenden wird beschrieben, wie eine Realexperimentreihe realisiert werden kann, wobei dafür auf die Erfahrungen mit DNE im Reallabor Karlsruhe zurückgegriffen wird. Abb. 1 veranschaulicht die verschiedenen Phasen und Tätigkeiten, die zur Durchführung einer solchen Realexperimentreihe notwendig sind. In jeder dieser Phasen haben sich im Falle von DNE eine Methode oder ein Methodenkoffer (zusammenhängendes Set verschiedener Methoden) als besonders hilfreich erwiesen. Daher werden diese im folgenden Text detaillierter vorgestellt. Der Begriff Methode umfasst in diesem Fall sowohl wissenschaftliche Forschungsmethoden als auch kommunikative und interaktive Vorgehensweisen zur Gestaltung des Dialogs aus Moderationspraxis und Kommunikationsdesign. Das Ziel dieser Methoden sind sowohl Erkenntnisgewinn (Wissensproduktion) als auch gesellschaftliche Intervention.

Bei der Darstellung des Vorgehens wird besonderes Gewicht gelegt auf die Initiierungsphase (v. a. Partnerworkshop) und die Aufbauphase (Ansprache der Akteure und Wettbewerb). Mit Blick auf die Durchführungsphase eröffnet sich mit dem Methodenkoffer 2 ein Einblick insbesondere in die wissenschaftlichen Vor-

4 Für ausführliche Beschreibungen der Nachhaltigkeitsexperimente siehe http://www. quartierzukunft.de 


\begin{tabular}{|c|c|c|}
\hline $\begin{array}{l}\text { Initiierungsphase } \\
1-3 \text { Monate }\end{array}$ & $\begin{array}{l}\text { Tätigkeiten } \\
\text { - Kooperationsaufbau } \\
\text { - Themenwahl }\end{array}$ & $\begin{array}{l}\text { Methoden } \\
\text { - Partnerworkshop }\end{array}$ \\
\hline $\begin{array}{l}\text { Aufbauphase } \\
6 \text { Monate }\end{array}$ & $\begin{array}{l}\text { - Planung und Durchführung } \\
\text { eines Wettbewerbs } \\
\text { - Vorbereitung der wissen- } \\
\text { schaftlichen Begleitforschung }\end{array}$ & $\begin{array}{l}\text { Methodenkoffer } 1 \\
\text { - Alltagsnahe Sprachfindung } \\
\text { - Presse- und Medienarbeit } \\
\text { - Corporate Design \& Logo }\end{array}$ \\
\hline $\begin{array}{l}\text { Durchführungs } \\
\text { phase } \\
9-12 \text { Monate }\end{array}$ & $\begin{array}{l}\text { - Durchführung der } \\
\text { Realexperimente } \\
\text { - Durchführung der } \\
\text { Begleitforschung }\end{array}$ & $\begin{array}{l}\text { Methodenkoffer } 2 \\
\text { - Beforschung } \\
\text { - Betreuung } \\
\text { - Begleitung }\end{array}$ \\
\hline $\begin{array}{l}\text { Auswertungs- } \\
\text { phase } \\
6-12 \text { Monate }\end{array}$ & $\begin{array}{l}\text { - Erfassung des Gesamtmaterials } \\
\text { - Transkription und Überführung in } \\
\text { auswertbare Formen } \\
\text { - Auswertung } \\
\text { - Veröffentlichungen }\end{array}$ & $\begin{array}{l}\text { - Datensichtungsworkshop } \\
-\ldots \\
-\ldots\end{array}$ \\
\hline
\end{tabular}

Abbildung 1: Schematischer Aufbau einer Realexperimentreihe (schwarz unterlegt die Phasen, auf die im Beitrag eingegangen wird). (c) Quartier Zukunft, R131.

gehensweisen während der Experimentlaufzeit. Nicht thematisiert wird im vorliegenden Beitrag die sich daran anschließende Auswertungsphase (die im Falle von DNE zum Zeitpunkt des Abschlusses des Manuskripts noch am Laufen war).

Die vorgestellten Methoden entstanden aus den Erfahrungen des Reallabors Karlsruhe bei Aufbau und Durchführung der Experimentreihe „Dein NachhaltigkeitsExperiment“ (DNE). Sie sollen als Anregung zum Nachmachen dienen; eine Anpassung kann und sollte bei abweichenden Grundvoraussetzungen (etwa kürzerem Zeitraum) vorgenommen werden.

\subsection{Initiierungsphase: Kooperationsaufbau durch einen Partnerworkshop}

Die Initiierungsphase dient der Themenfindung und ggf. Findung von Kooperationspartnern zur Durchführung einer Realexperimentreihe. 
Bei der Konzipierung und späteren Durchführung gemeinsamer Realexperimente sind alle Beteiligten vor die Herausforderung gestellt, unterschiedliche Interessen, Arbeitsweisen, Disziplinen, (Fach-)Sprachen und Werthaltungen zu einem gemeinsamen Ganzen zu vereinen. Das Finden eines gemeinsamen Verständnisses grundlegender Begriffe (im Falle von DNE Nachhaltigkeit, Gemeinschaft, Entschleunigung, Experiment) und die Einigung auf gemeinsame Ziele und Vorgehensweisen im Experimentprozess sollten so früh wie im gegebenen Setting möglich erfolgen. Der Entwicklung gemeinsamer Ziele und der Vereinbarung eines konsistenten Vorgehens ist besonderes Augenmerk zu schenken: Bei der Dynamik, die Realexperimente entwickeln können, weil sie in realweltlichen Kontexten durchgeführt werden, ist es ratsam, Kooperationen auf eine stabile und vertrauensvolle Basis des Miteinanders zu stellen. Mitunter müssen die Projekte auch im Verlauf rejustiert und an Entwicklungen angepasst werden. Dies erfordert ein schnelles (Re-)Agieren und Entscheiden, das durch einmalige Absprachen oder Kooperationsvereinbarungen zu Beginn nicht abgedeckt werden kann. Eine solide gemeinsame Basis der Kooperationspartner ist hierfür essentiell. Dies bedeutet zum einen, dass die Kooperationspartner sich kennen und schätzen (zumindest respektieren und akzeptieren), und zum anderen, dass sie ein Grundverständnis für die Arbeitsweise, Sprache, Werthaltungen und Interessen der Anderen haben.

Aufgrund enger Zeitpläne wird zu Projektbeginn oft versucht, mit einem neuen Kooperationspartner gleich in die thematische Arbeit einzusteigen. Eine vorangehende Phase des Kennenlernens wird meist vernachlässigt. Sie stellt jedoch das Fundament für eine gute Zusammenarbeit dar (für weiterführende Literatur zu Prinzipien transdisziplinärer Zusammenarbeit im Forschungsalltag s. Di Giulio et al. 2016) und darf insbesondere bei einer Realexperimentreihe nicht zu kurz kommen. Der im Folgenden beschriebene Partnerworkshop ist eine Möglichkeit, eine solche gemeinsame Arbeitsbasis aufzubauen, und steht dementsprechend am Anfang einer Kooperation.

Der nachstehend beschriebene Ablauf kann in Bezug auf die einzelnen Schritte und Methoden variieren. Ein solcher Partnerworkshop dauert 1 bis 1,5 Tage, je nachdem, wie viel Zeit am Stück genutzt werden kann und wie viele Partner involviert sind (mindestens erforderlich sind jedoch 6 Stunden exklusive Pausen). Wird er aufgeteilt, sollte zwischen den einzelnen Teilen kein zu großer zeitlicher Abstand liegen. Sind mehrere Kooperationspartner involviert, sollten Vertreter(innen) aller Partner teilnehmen. Der hier beschriebene Ablauf setzt voraus, dass die thematische Ausrichtung bereits feststeht. Wollen Partner zusammen eine Realexperimentreihe initiieren, wissen aber noch nicht, zu welchen Themen, sollte ein anderer Startpunkt (etwa eine Ideenwerkstatt o. Ä.) gewählt werden. Da die Wissenschaftler(innen) ebenfalls am Workshop teilnehmen, ist es ratsam, eine externe Moderation hinzuzuziehen. Sollte dies nicht möglich sein, so ist aus dem 
Team zumindest eine Person mit Moderationserfahrung zu wählen. Der Prozess ist so offen und transparent wie möglich zu gestalten und in allen Fällen in geeigneter Weise zu dokumentieren.

\subsubsection{Kennenlernen (Dauer 30 Minuten bis 2 Stunden)}

Um das Kennenlernen und eine gegenseitige Verständigung einzuleiten, ist es hilfreich, mit Kommunikationsübungen und Kennlernaktivitäten einzusteigen. Folgende Methoden sind für Gruppen von 4 bis 15 Personen gut geeignet:

Die Methode des Aktiven Zuhörens kann eingesetzt werden, um einzelne Personen im 2er-Gespräch besser kennenzulernen. Diese Methode dauert je nach Teilnehmerzahl etwa 20-40 Minuten. Es ist darauf zu achten, dass sich möglichst Paare zusammenfinden, die sich noch nicht gut kennen. Aktives Zuhören ist eine Basismethode der Moderation und kann in vielen Kontexten angewendet werden. Es bedeutet, nicht einfach abzuwarten, bis die andere Person zu Ende gesprochen hat, sondern zuzuhören, ohne gleich an eine Gegenrede zu denken oder diese zu formulieren.

„Aktives Zuhören bedeutet darüber hinaus, eine interessierte Haltung einzunehmen und den Gesprächspartnern äußerlich und innerlich zugewandt zu sein. Wem so zugehört wird, der fühlt sich eher verstanden und akzeptiert, braucht sich nicht ständig zu wiederholen und ist eher bereit, sich die andere Seite anzuhören." (Werkstatt für Gewaltfreie Aktion Baden 2004, S. 186).

Im Falle von DNE fanden sich zu Beginn des Partnerworkshops mit der Bürgerstiftung Karlsruhe die Teilnehmer(innen) dazu in Kleingruppen von zwei Personen zusammen (möglichst gemischte Tandems, z. B. Wissenschaftler(in) und Person aus Bürgerstiftung) und stellten sich dem Gegenüber in etwa fünf Minuten kurz vor. Anschließend stellte die jeweils andere Person ihn/sie der Gruppe vor, z. B.: „Ich beschreibe Frau X von der Bürgerstiftung. ...“.

Eine weitere Möglichkeit des gegenseitigen Kennenlernens, die mehr die gesamte Gruppe in den Blick nimmt, ist die Zahlenstrahl-Methode. Diese Methode dauert 20-45 Minuten (je nach Teilnehmer(innen)zahl).

„Dabei wird durch Aufstellungen im Raum das Spektrum der Standpunkte im Raum gut sichtbar [...]. Außerdem kann die Bewegung eine erfrischende Abwechslung sein und Leute aus ihren erstarrten Sichtweisen bringen“ (Werkstatt für Gewaltfreie Aktion Baden 2004, S. 187).

Die Anwesenden werden gebeten, sich zu bestimmten Aussagen im Raum zu positionieren. Dabei bildet der Strahl eine Skala oder ein Kontinuum. Anfang und Ende des Strahls markieren jeweils zwei Gegenpole einer Aussage, Meinung oder Antwort. Mögliche Fragen sind: Wie viele Leute sehe ich hier zum ersten Mal? Wie stark verbunden fühle ich mich mit dem Thema X (im Falle von DNE: Nachhaltigkeit)? Als weiteren Schritt kann die Moderation bei Personen auf dem 
Strahl/auf der Skala rückfragen, was die Beweggründe für ihre Positionierung sind. Dabei erfolgen jedoch keine Diskussionen und Bewertungen.

Im Anschluss an das Kennenlernen empfiehlt sich eine kurze Pause, nach der die themenbezogene Arbeit beginnt.

\subsubsection{Sichtweisen auf das Thema sammeln (Dauer: 2 bis 4 Stunden)}

Mit Blick auf die Klärung zentraler Begriffe und der Entwicklung eines gemeinsamen Verständnisses solcher Begriffe werden in moderierter Kleingruppenarbeit die individuell unterschiedlichen Verständnisse dieser Begriffe (optimal 3-5 Stück) zunächst sichtbar gemacht. Dies geschieht durch eine nicht bewertende Sammlung der mit einem Begriff assoziierten Stichworte. Dazu finden sich die Teilnehmer(innen) in Gruppen zusammen, und jede Gruppe arbeitet an einem für das Thema der geplanten Zusammenarbeit zentralen Begriff (im Falle von DNE waren leitende Fragen dafür: Was gehört für uns zum Begriff Gemeinschaft bzw. Entschleunigung? Ist uns eine Abgrenzung zu einem anderen Begriff wichtig?). Die Gruppen sollten möglichst gleichgewichtet mit Angehörigen aller Partner (also inkl. Wissenschaft) besetzt sein. Die Stichworte werden auf Moderationskarten gesammelt. Diese Art der Abfrage bietet viele Vorteile, sie

„gibt jedem Gruppenmitglied eine gleiche Beteiligungschance und führt in kürzester Zeit zu einem umfangreichen Pool an Ideen und Vorschlägen. Die Einzelarbeit beim Ausfüllen der Kärtchen stellt sicher, dass jede(r) unbeeinflusst von den anderen Gruppenmitgliedern ihre/seine Gedanken entwickelt und zu Papier bringt“" (Werkstatt für Gewaltfreie Aktion Baden 2004, S. 195).

Nachdem die Moderation die Karten eingesammelt hat, werden die Begriffe geclustert (im Fall von DNE waren die Cluster Ziele/Voraussetzungen, Abgrenzung, Herausforderung und Weg, s. Abb. 2). Dabei werden die gesammelten Karten nach Kategorien geordnet, die sich aus den gesammelten Stichworten ableiten. Alle Teilnehmenden haben anschließend die Möglichkeit, zu einzelnen Stichworten Rückfragen zu stellen und falls nötig zu ergänzen oder zu konkretisieren. Das Ziel ist hier, die Sichtweise der Anderen auf das gemeinsame Thema kennenzulernen, Gemeinsamkeiten und Unterschiede zu erkennen und eine gemeinsame begriffliche Basis zu etablieren.

Dies ist der erste Schritt für ein geteiltes Begriffsverständnis. Die Arbeit in Kleingruppen dient nicht nur der Arbeitseffizienz, sondern vor allem dem intensiveren Austausch und Kennenlernen sowie der Aktivierung aller Beteiligten. Ist die Gruppe zu groß, können sich meist nicht alle aktiv beteiligen, was Unmut erzeugen kann. Auch trauen sich stille und zurückhaltende Personen oft nicht zu Wort. Durch die Arbeit in Kleingruppen entsteht teilweise ein höherer Zeitaufwand, die Ergebnisse sind aber zumeist für die Beteiligten zufriedenstellender 


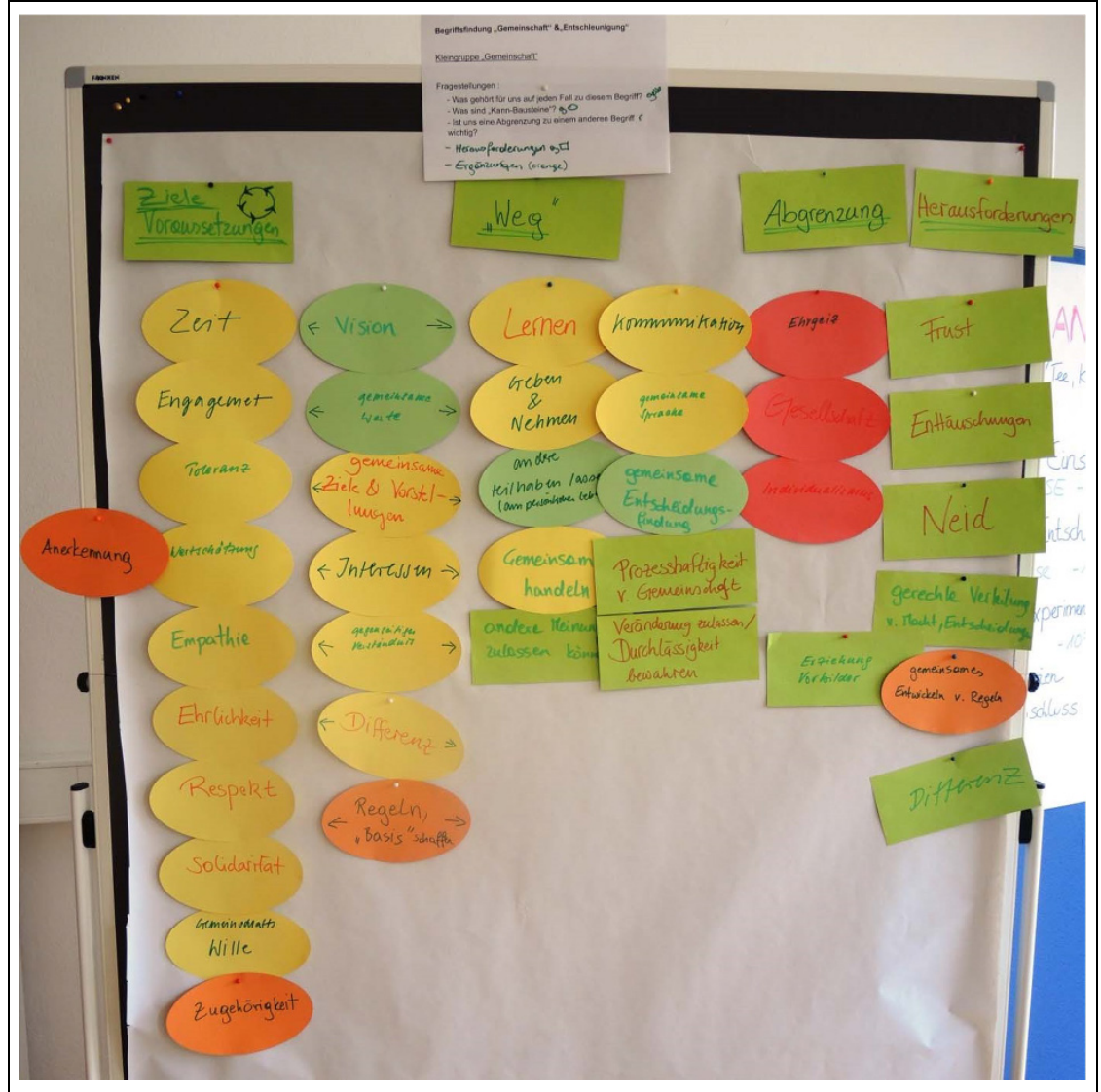

Abbildung 2: Darstellung der Clusterung des Partnerworkshops nach zentralen Begriffen. (C) Quartier Zukunft, R131.

und langfristig tragfähiger. Zudem beinhalten sie die Sichtweise aller Personen, und damit wird das Potential der gesamten Gruppe genutzt. In einer Gruppe arbeiten optimalerweise maximal sechs Personen.

\subsubsection{Gemeinsame Konkretisierung von Zielen und Themen (Dauer 2 bis 4 Stunden)}

Auf der Grundlage der so erfolgten begrifflichen Verständigung werden in einem nächsten Schritt die Wünsche an das gemeinsame Vorhaben offengelegt. In diesem Schritt kann es hilfreich sein, Teilnehmer(inne)n die Möglichkeit zu geben, zwischen ihrer persönlichen Ansicht und ihrem Standpunkt als Vertreter(in) einer 
Organisation/Institution zu unterscheiden (für die Aufgabenstellung im Falle von DNE s. Abb. 3), um ein breiteres Spektrum an Antworten zu erhalten. ${ }^{5}$ Auch hier erfolgt die Erarbeitung mit Moderationskarten, die anschließend im Plenum gruppiert werden. Persönliche Aussagen werden hierbei inhaltlich nicht diskutiert, die Interessen der Organisation/Institution jedoch in einer anschließenden Diskussionsrunde im Plenum erläutert. Im Falle von DNE wurden folgende Fragen diskutiert: Warum ist dies der jeweiligen Organisation wichtig? Können die Partner dem zustimmen oder müssen Anpassungen gemacht werden, damit alle Partner hier mitgehen können?

Ziel ist es, Gemeinsamkeiten zu identifizieren und einen Umgang mit potentiell divergierenden Interessen zu finden, um auf dieser Grundlage zu einer Einigung auf eine gemeinsame Vorgehensweise zu gelangen. In diesem Schritt muss nicht zwangsläufig ein umfänglicher Konsens auf allen Ebenen hergestellt werden. Jedoch sollte der vorangegangene Prozess zur Akzeptanz der unterschiedlichen Ansichten und Interessen führen und so die Türen für eine gemeinsame Vorgehensweise öffnen. Abb. 3 zeigt beispielhaft einzelne Schritte dieses Annäherungsprozesses im Falle von DNE.

Gegen Ende des Workshops werden die bisherigen Ergebnisse von der Moderation zusammengefasst. In einer anschließenden moderierten Plenumsdiskussion sollten folgende Fragen geklärt werden: Haben die Partner die gegenseitigen Interessen und Ziele verstanden? Gibt es Interessen, die einander zuwiderlaufen? Wenn ja: Wie wollen wir damit umgehen? Braucht es zur Klärung noch einen weiteren Workshop? Wo ergänzen wir uns gut? Besteht noch Uneinigkeit zwischen den Partnern, muss eine weitere Arbeitsschleife, etwa in Form eines weiteren Workshops, eingebaut werden. Falls Einigkeit erzielt ist, können in einem nächsten Schritt die Festlegung auf die zu verwendenden Kriterien für die Auswahl von Experiment-Ideen und die Festlegung der weiteren gemeinsamen Vorgehensweise erfolgen.

Das gemeinsam erarbeitete Material wird von der Moderation fotografisch festgehalten und in Form eines (Foto)Protokolls an alle Teilnehmenden verschickt. Es dient als Grundlage für die zukünftige Zusammenarbeit.

5 Hier ist das (inzwischen erarbeitete) Vertrauensverhältnis der Teilnehmer(innen) untereinander ausschlaggebend dafür, wie weit sich die Beteiligten trauen, ihre von der organisationalen/institutionellen Sicht ggf. abweichenden persönlichen Ansichten zu äußern. Es kann durchaus sinnvoll sein, bestimmte Teile des Partnerworkshops als ,vertraulich“ zu bezeichnen und dies vorweg klarzustellen. 


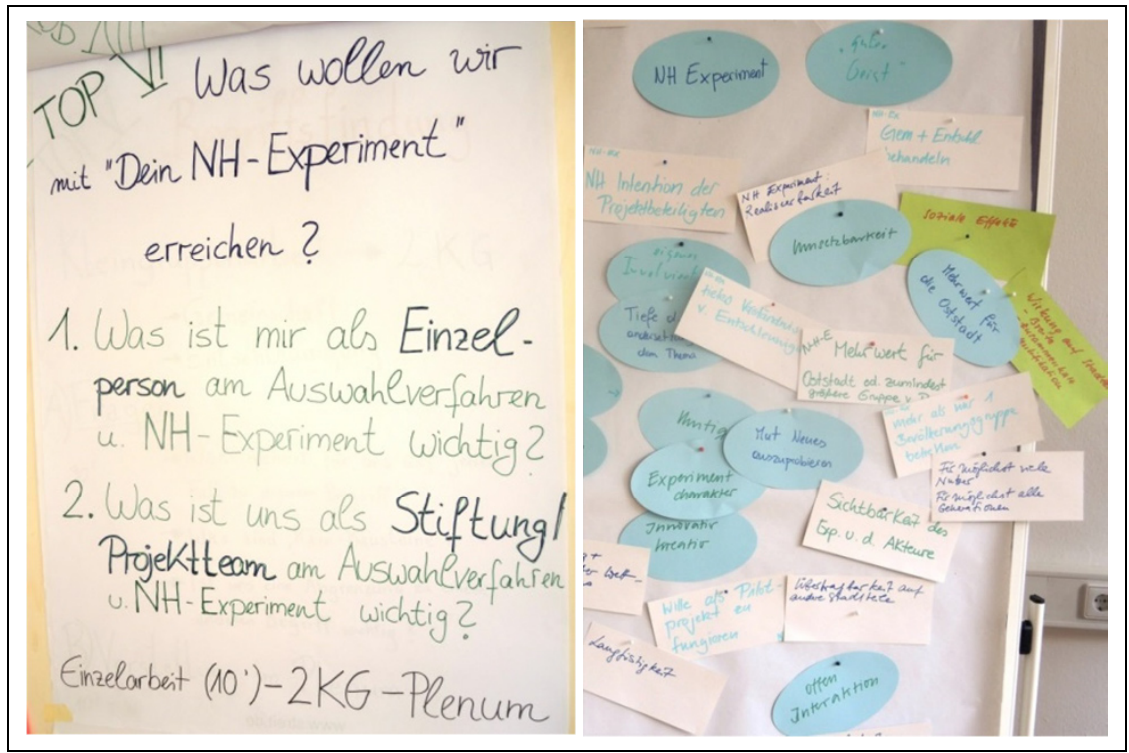

Abbildung 3: In der Kleingruppenarbeit werden Ziele und Wünsche erfragt. Das zweite Foto zeigt einen Ausschnitt aus der Vielfalt der Antworten auf die erste Frage des ersten Fotos. (C) Quartier Zukunft, R131.

\subsubsection{Reflexion (30 Minuten bis 1 Stunde)}

Zum Schluss sollte noch eine von der inhaltlichen Arbeit entkoppelte Feedbackrunde stattfinden, die dazu dient, festzustellen, wie sich die Teilnehmenden fühlen, und darauf abzielt, das Gemeinschaftsgefühl der Gruppe zu stärken. Ein solches Stimmungsbild kann über verschiedene Methoden erhoben werden, etwa über ein Blitzlicht. Reihum haben hier alle Teilnehmer(innen) die Möglichkeit, zu einem bestimmten Thema ein Statement abzugeben. So kann etwa gefragt werden, wie sich die Teilnehmer(innen) nun zum Workshopende fühlen und was sie an der bisherigen Zusammenarbeit gut und was weniger gut fanden, aber auch, worauf sie sich freuen. Jede(r) entscheidet jedoch selbst, ob er/sie etwas sagen möchte. Die Statements bleiben unkommentiert. Ein solches Blitzlicht dauert je nach Gruppengröße und Redebereitschaft 10 bis 30 Minuten.

Eine weitere, etwas verspieltere Variante der Stimmungsabfrage, stellt die Methode Blumenwiese dar. Diese Methode dient dazu, dem Abschluss einer Veranstaltung eine konstruktiv-positive Einleitung zu geben. Hierzu werden allen Teilnehmer(inne)n Fragen gestellt, deren Antworten im Anschluss auf aus Papier ausgeschnittene Blumen geschrieben und auf einer grünen Decke/einem grünen Poster (symbolisiert die Wiese) aufgesteckt oder aufgeklebt werden. Damit auf 
die Aussagen in der weiteren Zusammenarbeit zurückgegriffen werden kann, empfiehlt es sich, die erarbeitete Blumenwiese gut sichtbar in den auch in $\mathrm{Zu}$ kunft genutzten Räumlichkeiten (falls es solche gibt) aufzuhängen. Mögliche Fragen für die Blumenwiese sind: Auf was sollten wir in unserer Zusammenarbeit besonders achten? Was gefällt mir an unserer heutigen und bisherigen Zusammenarbeit? Was ist mir sonst noch wichtig mitzuteilen?

\subsection{Aufbauphase: Aktivierung und Ansprache der Akteure und Vorbereitung der Experimente}

Ist die Zusammenarbeit mit den Kooperationspartnern auf eine stabile Basis gestellt, besteht die nächste zentrale Aufgabe in der Aktivierung und Einbindung von Gruppen von Bürger(inne)n, welche in der Folge die Realexperimente entwickeln und durchführen werden. Die Frage, wie solche Akteure angesprochen und zur aktiven Teilnahme motiviert werden können, ist für das Gelingen einer Realexperimentreihe zentral. Im Folgenden werden jene Methoden vorgestellt, die sich dafür besonders anbieten.

\subsubsection{Ein Wettbewerb als Aktivierungsinstrument}

Ein Ideen-Wettbewerb stellt den außenwirksamen Auftakt einer Realexperimentreihe dar. Das Wettbewerbsformat schafft einen Raum, in dem Ideen generiert, an die Öffentlichkeit gebracht und ggf. umgesetzt werden können. Es eignet sich daher gut, um einen thematischen Impuls zu setzen, den Bürger(innen) durch eine Bewerbung aufgreifen und nach ihren Vorstellungen gestalten und umsetzen können (s. a. Puttrowait et al. 2018). Ziel des Wettbewerbs ist es, eine breite Spanne von Transformationsprozessen anzustoßen. Im Reallabor Karlsruhe sollte damit dem Ziel integrativer Nachhaltigkeit Rechnung getragen und gleichzeitig eine breite Basis für die Wissensproduktion geschaffen werden.

Bei der Auslobung des Wettbewerbs wird dazu aufgerufen, Ideen für Realexperimente einzureichen (Anlage a zeigt das Poster, welches im Falle von DNE als Ankündigung in Karlsruhe ausgehängt wurde). Ideen sollen aber nicht nur eingereicht, sondern nach dem Wettbewerb von den Einreichenden auch realisiert werden - bei Gewinn mit Unterstützung durch die Wettbewerbsprämien. Um die Teilnahme attraktiv zu machen, ist es wichtig, dass die Beteiligten einen Mehrwert aus dem Experiment ziehen können; sei es die Möglichkeit zur Umsetzung ihrer Ideen, Medienwirksamkeit für eigene Anliegen oder eine finanzielle Förderung. Der Bewerbungsprozess sollte dabei so niederschwellig und unbürokratisch wie möglich gehalten werden. Die Teilnahmebedingungen und das Bewerbungsformular sollten deshalb online zur Verfügung gestellt werden, bei Bedarf aber auch in gedruckter Form abgeholt werden können. Im Bewerbungsformular sollten Idee und Motivation in sehr freier Form beschrieben werden können, für den 
vorläufigen Zeit- und Finanzplan bietet es sich an, eine Tabelle zur Verfügung zu stellen, die aber nach Bedarf adaptiert werden kann. Die Teilnahmebedingungen enthalten neben den konkreten Anforderungen auch eine Übersicht über das Bewerbungs- und Auswahlprozedere (für die Unterlagen, die im Falle von DNE verwendet wurden, s. Anlage b). Die Anzahl der geförderten Experimente sowie Kriterien der Auswahl und das Auswahlverfahren sind mit den Kooperationspartnern festzulegen und sind meist abhängig von den finanziellen Mitteln und der thematischen Ausrichtung der Partnerinstitutionen. Im Falle von DNE haben sich die Partner bewusst dafür entschieden, mehrere eingereichte Experimentideen zu prämieren. So wurden das inhärente Konkurrenzmoment eines Wettbewerbs abgeschwächt und die Möglichkeit geschaffen, unterschiedliche Ideen parallel umsetzen und auswerten zu können. Sinnvollerweise sollte aber mit dem Wettbewerb im Detail das praktiziert werden, worauf Reallabore und Realexperimente im Großen abzielen: die Kooperation von Akteuren aus Wissenschaft und Zivilgesellschaft. Obwohl die genaue Ausgestaltung des Auswahlprozesses je nach Partnerkonstellation variiert (und daher im Folgenden nicht weiter vertieft wird), sollte darauf hingewirkt werden, dass die Projektauswahl gemeinsam erfolgt und dass die Kooperationspartner mindestens einen Teil des Preisgeldes zur Verfügung stellen. Es empfiehlt sich, zur Planung des Auswahlverfahrens einen Workshop abzuhalten (ggf. angelehnt an den Partnerworkshop).

Die Durchführung des Wettbewerbs erfordert eine intensive Vorbereitung und kann in folgende Schritte unterteilt werden.

Vorbereitung (4-6 Monate): Thema und Ziel für die Realexperimentreihe werden ausgearbeitet. Die Inhalte werden grafisch und textlich aufbereitet und in verschiedene Formate überführt (Plakate, Kurzfilm, Postkarte, Blogbeiträge etc.). Prozessschritte und Zeitrahmen für den Wettbewerb werden (gemeinsam mit den Kooperationspartnern) erarbeitet.

Durchführung (2-3 Monate): Der Wettbewerb wird öffentlichkeitswirksam ausgerufen, indem die zuvor erarbeiteten Werbeformate veröffentlicht werden. Auch die Presse sollte einbezogen werden. Der Bewerbungszeitraum sollte 8 Wochen nicht unterschreiten.

Juryarbeit (1-2 Wochen): Eine mehrköpfige Jury wird gebildet. Sind mehrere Kooperationspartner an der Realexperimentreihe beteiligt, sollte das Verhältnis in der Jury ausgewogen sein. Die Jurymitglieder verständigen sich in einem gemeinsamen Prozess vorab auf die Art und Weise der Entscheidungsfindung und erarbeiten Kriterien zur Bewertung der Bewerbungen. Empfehlenswert ist ein zweistufiges Verfahren, in dem einige vielversprechende Gruppen eingeladen werden, ihre Ideen noch einmal persönlich der Jury vorzustellen.

Bekanntgabe der Gewinner: Die Bekanntgabe der Gewinner erfolgt schriftlich (per E-Mail oder Brief), danach kann auch die Presse einbezogen werden. Gibt es 
eine Preisverleihung, sollten alle Bewerber(innen) eingeladen und auch die nicht ausgewählten Beiträge gewürdigt werden. In der Folge sollten in jedem Fall bindende Verträge mit den Preisträgern zur zweckbestimmten Verwendung der Preisgelder und zur Teilnahme am Realexperiment abgeschlossen werden.

\subsubsection{Methodenkoffer 1: Werkzeuge einer adäquaten Kommunikation}

Für den Wettbewerb, aber auch für den nachfolgenden Experimentzeitraum, ist die passende Art der Kommunikation ein wichtiger Erfolgsfaktor für die Realexperimentreihe. Im Folgenden wird daher ein Set an Methoden zur (Außen-) Kommunikation vorgestellt.

\section{Passende (An-)Sprache der Akteure}

Vor dem Start einer Realexperimentreihe ist es wichtig, die Zielgruppe der Realexperimente, also die Menschen, die ihre Ideen einreichen sollen, zu definieren, sich gedanklich in diese hineinzuversetzen und eine dementsprechend adäquate Strategie zur Außenkommunikation festzulegen. Eine alltagsnahe Sprache, die realweltliche Bezüge zu den Themen der Realexperimentreihe (im Falle von DNE also zu Gemeinschaft und Entschleunigung) herstellt, und erlaubt, komplexe Sachverhalte für die Zielgruppe interessant und ansprechend darzustellen, bildet dafür die Kommunikationsbasis. Es geht vor allem darum, das Interesse für das Thema zu wecken; Belehrungs-Charakter sollte vermieden werden.

\section{Sichtbarkeit und Wiedererkennungswert sicherstellen}

Um bei Bürger(inne)n Interesse zu wecken und dieses auch in der Durchführungsphase der Realexperimente zu erhalten, sollten die gewählten Kommunikationswege klar durchdacht werden, und es sollten aufeinander aufbauende Inhalte kommuniziert werden. Um die Sichtbarkeit zu erhöhen, ist es sinnvoll und wirksam, eine Bandbreite an verschiedenen Medienkanälen abzudecken. Es sollten also unterschiedliche Formate der Werbung kombiniert werden: Plakate, Ankündigungen über die sozialen Medien und in Zeitungen, Zeitschriften- und Blogbeiträge, Kurzfilme, Postkarten. Es gilt, die Kanäle und Formen der Werbung der Zielgruppe und der lokalen Medienlandschaft entsprechend klug zu wählen. Um den Wiedererkennungswert der Realexperimentreihe zu erhöhen, ist es nützlich, zu Beginn ein Logo oder Motive zu etablieren, die sich dann auf allen Werbemitteln wiederfinden (Abb. 4 zeigt, wie sich im Falle von DNE die Charaktere der Kurzfilme auf den Postkarten und den Fensterscheiben des Quartiersbüros Zukunftsraum wiederfinden). 

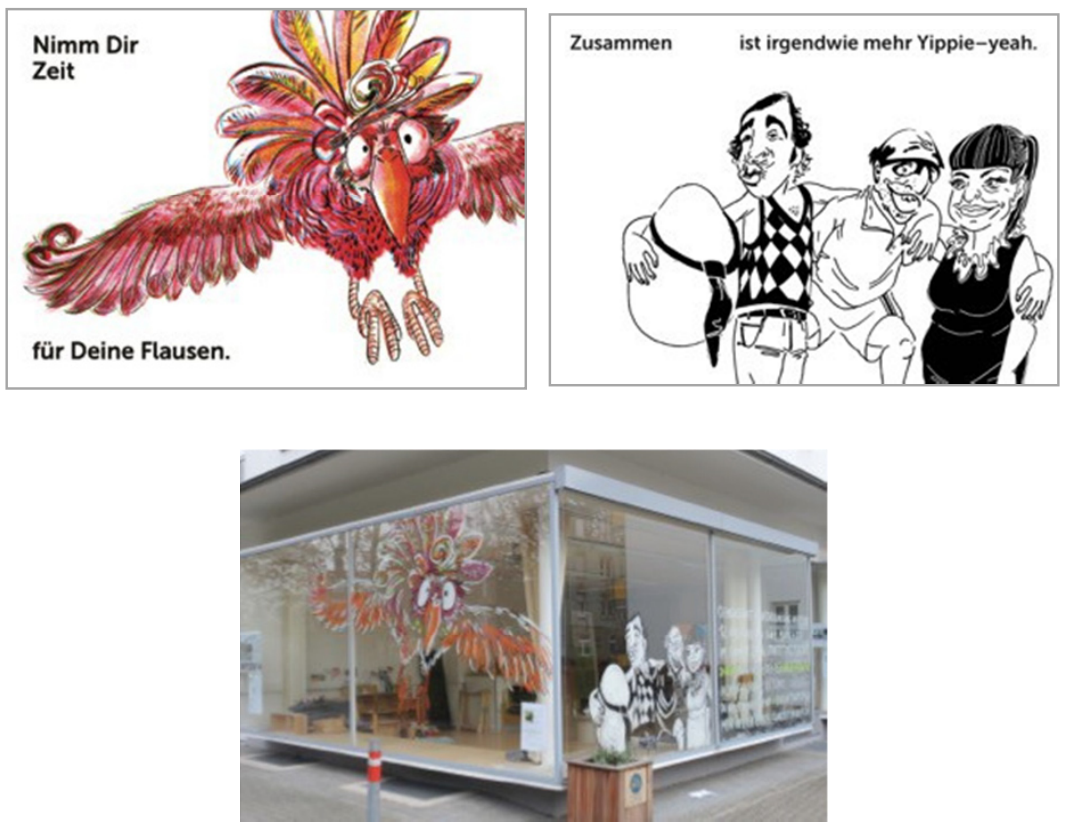

Abbildung 4: Bestimmte Charaktere fanden sich sowohl in den Kurzfilmen als auch auf den Postkarten zu DNE und dem Schaufenster des Zukunftsraumes des Reallabors Karlsruhe wieder. So wurde ein Wiedererkennungswert erzeugt. (C) Quartier Zukunft, R131.

\section{Kurzfilme für die Werbung}

Eine prüfenswerte, wenn auch anspruchsvolle Möglichkeit besteht darin, die Realexperimentreihe mittels Kurzfilmen zu bewerben. Kurzfilme können mit Original-Ton der Darsteller(innen) oder einer Erzählstimme ausgestattet sein; aber auch Stummfilme, die nur über Schrift und Toneffekte arbeiten, sind möglich. Für die Erstellung bedarf es einer mit Filmschnitt und Grafik erfahrenen Person. Die Formulierung eines Storyboards zu Beginn ist essentiell. Nicht zu unterschätzen ist der Zeitaufwand für die Erstellung von Animationen oder Trickfilmsequenzen, je nach Erfahrungsgrad der ausführenden Personen sollten hier mindestens 6-8 Wochen eingeplant werden. Ein Kurzfilm sollte 2-4 Minuten nicht überschreiten. Für die Bewerbung von DNE wurden u. a. zwei Kurzfilme geschaffen, um die Inhalte exemplarisch aufzubereiten und unterhaltsam darzustellen (Abb. 5 zeigt Ausschnitte aus einem der beiden Kurzfilme zur Bewerbung des Wettbewerbs). 


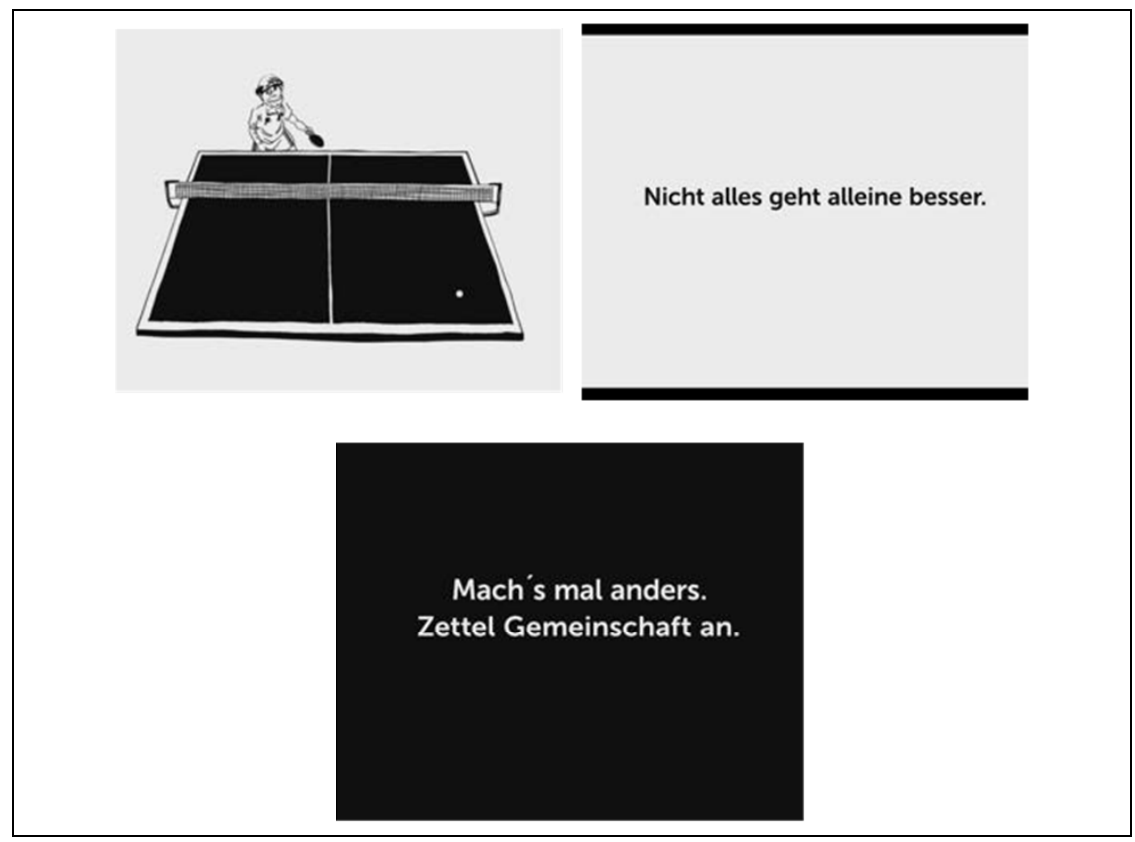

Abbildung 5: Kurzfilm „Wer will schon alleine sein?“ zu Gemeinschaft. Im Falle von DNE ging es darum, mit kreativen Szenen den Wert von Gemeinschaft nahezubringen, der Abspann sollte neugierig auf die Realexperimente machen und zum Mitmachen animieren. (C) Quartier Zukunft, R131.

\subsection{Durchführungsphase}

Die Durchführung und gleichzeitige Beforschung einer neunmonatigen Realexperimentreihe ist ein in mehrfacher Hinsicht komplexes Unterfangen. Zum einen finden die Experimente parallel statt, was Herausforderungen an Planung, Logistik und eingesetzte Arbeitskapazitäten mit sich bringt. Zum anderen erfordern die mit dem Format verbundenen multiplen Zielsetzungen (vgl. für DNE Abschnitt 1.2) zeitgleich unterschiedliche wissenschaftliche und organisatorische Tätigkeiten, die in der Regel in Personalunion durch die Wissenschaftler(innen) bewerkstelligt werden müssen. ${ }^{6}$

6 Je nach Partnerkonstellation können diese Aufgaben auch stärker zwischen den verschiedenen Partnern (Wissenschaft und Kooperationspartner) aufgeteilt werden. Im Falle von DNE war der Kooperationspartner nur in der Wettbewerbsphase und monetär eingebunden. 


\subsubsection{Methodenkoffer 2: Die vier B's der Projektarbeit: Beforschung, Betreuung, Begleitung ${ }^{2}$}

Bei der Durchführung von Realexperimenten greifen insbesondere die zeitlich und inhaltlich intensive Beforschung, die organisatorische Betreuung sowie die Begleitung der Experimentgruppen und des Reallabor-Teams ineinander. Diese Tätigkeiten sollten mit Blick auf eine gelingende Durchführung von Realexperimenten als Einheit konzipiert und miteinander verschränkt vollzogen werden. Ein für diese Phase der Realexperimentreihe geeigneter umfassender Methodenkoffer, der im Falle von DNE im Reallabor Karlsruhe eingesetzt wurde, wird im Folgenden dargestellt.

\section{Beforschung}

Die Beforschung (nicht nur auf der thematischen Ebene, sondern ggf. auch auf einer Meta-Ebene) speist sich aus zwei Quellen, wie Tabelle 1 veranschaulicht: Aus einem Set an Befragungen seitens der Wissenschaftler(innen), die sich an die Experimentgruppen richten, und aus der Selbstdokumentation der einzelnen Experimentgruppen. Die Beforschung fokussiert also auf die Personen, die zuständig sind für die Durchführung der Realexperimente (die Experimentierenden), während die darüber hinaus als Teilnehmer(innen) an den Veranstaltungen und Aktivitäten beteiligten Personen nur indirekt (etwa über die Veranstaltungsdokumentationen) erfasst werden.

Die Zweiteilung der Quellen wird gewählt, um eine große Bandbreite an Daten und damit einen bestmöglichen Einblick in die Gruppen- und Experimentprozesse zu erhalten. Die Außenperspektive der befragenden Wissenschaftler(innen) wird durch eine direkte Innenperspektive der Experimentierenden ergänzt. Die Auswahl der in den beiden Quellen eingesetzten Dokumentations- und Befragungsmethoden sowie deren Umfang, Intensität und Inhalt erfolgen durch die Wissenschaftler(innen), zugeschnitten auf die Zielsetzung und Funktionsweise der Experimente.

Tabelle 1: Die zwei Quellen der Beforschung in einer Realexperimentreihe.

\begin{tabular}{|l|l|}
\hline $\begin{array}{l}\text { Befragung \& Beobachtung } \\
\text { (Wissenschaftler(innen)) }\end{array}$ & $\begin{array}{l}\text { Selbstdokumentation } \\
\text { (Experimentgruppen) }\end{array}$ \\
\hline Fragebögen & Veranstaltungsdokumentation o. Ä. \\
Gruppeninterviews & Protokolle \\
Fokusgruppe & Fotodokumentation \\
& Rückblick \\
\hline
\end{tabular}


Durch ein transparentes Forschungsdesign und die Einbindung der Experimentgruppen in den Prozess der Datengewinnung wird die Reflexivität erhöht. So werden aber auch (transdisziplinäre) Lernprozesse aller Beteiligten gefördert, die nicht nur den Gegenstand des Realexperiments, sondern auch die eigene Rolle im Transformations- und Forschungsprozess umfassen. Die begleitende Forschung hat hier eine didaktische Funktion, sie soll Lernprozesse initiieren und inhaltlich bereichern, sie aber nicht steuern. Sie dient nicht allein der Produktion wissenschaftlicher Erkenntnisse, sondern durch den starken Einbezug der Experimentierenden und der Ausrichtung an deren Zielen auch diesen. Aufgabe ist es also, Forschungsmethoden zu finden und zu kombinieren, die sowohl die erforderlichen quantitativen und qualitativen Daten liefern als auch die Bürger(innen) bei der Durchführung und Reflexion ihrer Experimente unterstützen. So werden etwa über die Forschung Impulse gesetzt, sich intensiv mit dem eigenen Handeln und dem der Experimentgruppe auseinanderzusetzen. Im Falle von DNE wurde durch die Veranstaltungsdokumentation u. a. abgefragt, ob das Ziel der Veranstaltung erreicht wurde und falls nicht, aus welchen Gründen. Formate wie die Fokusgruppe oder wiederkehrende Gruppentreffen aller Experimentgruppen dienen zudem dazu, sich auszutauschen, zu vernetzen und Synergien zu nutzen. Im Folgenden werden die beiden Quellen der Beforschung genauer beschrieben.

a) Befragung durch die Wissenschaftler(innen):

- Mehrteilige Fragebogenerhebung (Mayer 2013; Porst 2014): Thematisch aufeinander aufbauende Fragebögen dienen der Erkenntnis zu den übergeordneten Themen der Realexperimentreihe (im Falle von DNE: Nachhaltigkeit, Gemeinschaft und Entschleunigung) sowie zu den Gruppenprozessen. Hier können einmalige Fragen, aber auch aufeinander aufbauende Fragegruppen zum Aufzeigen möglicher Veränderungen und Lernprozesse im Experimentverlauf formuliert werden. Jeder Fragebogen erhält einen anonymisierten Code. Diese Pseudonymisierung ermöglicht eine anonyme, vergleichende Auswertung der Fragebögen. ${ }^{7}$

- Gruppeninterviews (Helfferich 2014; Mayer 2013): Gruppeninterviews werden mit jeder einzelnen Experimentgruppe geführt, die Basis hierfür bildet ein Leitfaden. Die Interviews dienen dazu, die Einzelmeinungen aus der Fragebogenerhebung zu ergänzen und ggf. verschiedene Gruppenmeinungen einzuholen. $\mathrm{Zu}$ empfehlen ist, ein Interview nach der Hälfte der Experimentlaufzeit und eines nach Abschluss der Realexperimente durchzuführen. Im Nachgang der Interviews ist es empfehlenswert, seitens der Wissenschaftler(innen) ein Atmosphärenprotokoll über das Interview an-

7 Je nach Teilnehmerzahl und Nähe der Wissenschaftler(innen) zur Gruppe kann die Anonymisierung ggf. nicht vollständig gewährleistet werden. 
zufertigen. Hier werden Fragen nach der allgemeinen Stimmung und nach möglichen Auffälligkeiten thematisiert.

- Fokusgruppe (Henseling et al. 2006): Die Fokusgruppe findet experimentübergreifend statt. Mindestens zwei Mitglieder aus jeder Experimentgruppe nehmen teil. Sie dient dazu, sich übergreifenden Fragestellungen zu nähern und einen Austausch über die verschiedenen Experimente hinweg zu ermöglichen. Es handelt sich um eine qualitative Forschungsmethode, mit der Motivationen und Handlungshintergründe der Befragten herausgearbeitet werden können.

b) Selbstdokumentation der Experimentgruppen:

- Veranstaltungsdokumentation: Die Veranstaltungsdokumentation erfolgt anhand eines vorgegebenen Leitfadens für alle öffentlichen Veranstaltungen der Experimentgruppen während der 9-monatigen Experimentphase (für die in DNE verwendete Vorlage dazu s. Anlage c). Sie dient der Dokumentation der Außenwirkung der Aktivitäten der einzelnen Experimentgruppen und der reflexiven Auseinandersetzung der Experimentierenden mit ihren eigenen Zielsetzungen. Falls in der Realexperimentreihe keine öffentlichen Veranstaltungen realisiert werden, ist die Dokumentation an das realisierte Format anzupassen.

- Protokolle: Die Experimentierenden protokollieren ihre Arbeitstreffen. Arbeitsschritte, Diskussionen, Planänderungen und Entscheidungen, aber auch interne Gruppenprozesse werden dokumentiert. Finden keine regelmäßigen Arbeitstreffen statt (etwa wenn die Gruppe sehr familiär ist und Entscheidungen oft nebenher getroffen werden), werden regelmäßig rückblickende Zusammenfassungen für einen bestimmten Zeitraum erstellt.

- Fotodokumentation: Die Experimentierenden dokumentieren alle ihre Veranstaltungen sowie wichtige Arbeitsschritte innerhalb des Experiments fotografisch.

- Rückblick: Der Rückblick ist ein Hilfsmittel, um die Ereignisse und Prozesse der Zeit, bevor die Beforschung einsetzte, also z. B. bevor die Experimentgruppen ausgewählt wurden, zu dokumentieren. Er hält die Motivation und Ideenfindung für den Wettbewerb, die Phase der Gruppenbildung, und die Anfangszeit der Experimentgruppen anhand von Leitfragen, die durch die Wissenschaftler(innen) vorgegeben werden, in Textform fest.

Tabelle 2 gibt einen Gesamtüberblick über die in einer Realexperimentreihe durchgeführten Methoden, wobei bei jeder Methode ausgeführt wird, welchem Zweck sie in besonderer Weise dienen kann, geordnet nach Forschungs- und Transformationszielen, ergänzt um erfahrungsbasierte Anmerkungen zu Nutzen und Anwendbarkeit. 
Tabelle 2: $\quad$ Überblick über die Methoden zur Erzeugung von Daten in der Beforschung der Realexperimentreihe. Diese eignen sich nicht nur für die themenbezogene Forschung, sondern ggf. auch für eine Forschung auf der Meta-Ebene.

\begin{tabular}{|c|c|c|c|}
\hline Methode & Forschungsziele & Transformationsziele & Nutzen und Anwendbarkeit \\
\hline $\begin{array}{l}\text { Frage- } \\
\text { bögen }\end{array}$ & $\begin{array}{l}\text { Sicht und Aussa- } \\
\text { gen der einzelnen } \\
\text { Teilnehmer(in- } \\
\text { nen) (zu Themen, } \\
\text { zum Prozess etc.) } \\
\text { isoliert und ano- } \\
\text { nym erhalten. }\end{array}$ & $\begin{array}{l}\text { Über die Beschäftigung } \\
\text { mit den Fragen soll das } \\
\text { Nachdenken über die } \\
\text { abgefragten Themen } \\
\text { angeregt werden. }\end{array}$ & $\begin{array}{l}\text { Quantitative und qualitative } \\
\text { Daten können abgefragt und } \\
\text { verglichen werden. } \\
\text { Durch die starke Involviert- } \\
\text { heit sehr gute Rücklaufquo- } \\
\text { ten. Der allein auszufüllende, } \\
\text { anonyme Bogen gibt indivi- } \\
\text { duelle Ansichten wieder. }\end{array}$ \\
\hline $\begin{array}{l}\text { Gruppen- } \\
\text { interviews }\end{array}$ & $\begin{array}{l}\text { Einblicke in die } \\
\text { internen Prozesse } \\
\text { der Experiment- } \\
\text { gruppen erhal- } \\
\text { ten. }\end{array}$ & $\begin{array}{l}\text { Stärkung der Gruppe } \\
\text { als Gemeinschaft. } \\
\text { Nachdenken über ein- } \\
\text { gebrachte Themen } \\
\text { anregen. }\end{array}$ & $\begin{array}{l}\text { Direkte, persönliche Inter- } \\
\text { aktion mit den Akteuren. Fle- } \\
\text { xible, situative Lenkung der } \\
\text { Themen, Gelegenheit, Per- } \\
\text { sönliches einzubringen. } \\
\text { In persönlichen Gesprächen } \\
\text { entsteht am meisten Offen- } \\
\text { heit und Dynamik. }\end{array}$ \\
\hline $\begin{array}{l}\text { Fokus- } \\
\text { gruppe }\end{array}$ & $\begin{array}{l}\text { Gruppen- und } \\
\text { Experiment über- } \\
\text { greifende The- } \\
\text { men abfragen } \\
\text { Gemeinsamkei- } \\
\text { ten/Unterschiede } \\
\text { herausarbeiten } \\
\text { und im Gespräch } \\
\text { vertiefen (Syn- } \\
\text { these bilden). }\end{array}$ & $\begin{array}{l}\text { Neue Ansätze und } \\
\text { Sichtweisen können } \\
\text { aus dem Gruppenge- } \\
\text { spräch heraus entste- } \\
\text { hen und sich in der } \\
\text { künftigen Arbeit im } \\
\text { Experiment nieder- } \\
\text { schlagen. }\end{array}$ & $\begin{array}{l}\text { Dialogisches Format. Direkt } \\
\text { und stark reflexiv. } \\
\text { Beispiele aus der eigenen } \\
\text { Lebenswelt und provokative } \\
\text { Thesen regen zur Diskussion } \\
\text { an. }\end{array}$ \\
\hline
\end{tabular}




\begin{tabular}{|c|c|c|c|}
\hline \multicolumn{4}{|c|}{ b) Methoden der Selbstdokumentation durch die Experimentgruppen } \\
\hline Methode & Forschungsziele & Transformationsziele & Nutzen und Anwendbarkeit \\
\hline $\begin{array}{l}\text { Veranstal- } \\
\text { tungs- } \\
\text { dokumen- } \\
\text { tation }\end{array}$ & $\begin{array}{l}\text { Einblick in (Über- } \\
\text { blick über) die } \\
\text { vielfältigen Ver- } \\
\text { anstaltungen. } \\
\text { Belegbarkeit } \\
\text { sicherstellen. }\end{array}$ & $\begin{array}{l}\text { Zur Reflexion der Ver- } \\
\text { anstaltung anregen. } \\
\text { Für künftige Veranstal- } \\
\text { tungen lernen. }\end{array}$ & $\begin{array}{l}\text { Erzeugt Vergleichbarkeit. } \\
\text { Einzelne Aspekte geraten } \\
\text { schnell in Vergessenheit. We- } \\
\text { sentlicher Baustein zur Doku- } \\
\text { mentation der Historie. }\end{array}$ \\
\hline Protokolle & $\begin{array}{l}\text { Prozessschritte } \\
\text { nachverfolgen } \\
\text { können. Grup- } \\
\text { peninterne Aus- } \\
\text { einandersetzun- } \\
\text { gen erkennen. }\end{array}$ & $\begin{array}{l}\text { Transparenz sicher- } \\
\text { stellen. Ernsthaftigkeit } \\
\text { der Unternehmung be- } \\
\text { tonen. }\end{array}$ & $\begin{array}{l}\text { Fördert Transparenz und } \\
\text { Struktur innerhalb der Grup- } \\
\text { pen. } \\
\text { Weithin bekanntes Medium, } \\
\text { das einfach zu handhaben ist. } \\
\text { Standardisierung nötig (und } \\
\text { schwierig). }\end{array}$ \\
\hline $\begin{array}{l}\text { Foto- } \\
\text { dokumen- } \\
\text { tation }\end{array}$ & $\begin{array}{l}\text { Momente, Set- } \\
\text { tings, Stimmun- } \\
\text { gen und Emotio- } \\
\text { nen festhalten. }\end{array}$ & $\begin{array}{l}\text { Identität und emotio- } \\
\text { nale Bindung mit Expe- } \\
\text { riment stärken. Öffent- } \\
\text { lichkeitsarbeit unter- } \\
\text { stützen. }\end{array}$ & $\begin{array}{l}\text { Erinnerungsstützen, schöne } \\
\text { Momente der/für die Betei- } \\
\text { ligten. } \\
\text { Einfach zu handhaben, wird } \\
\text { meist sowieso gemacht (kein } \\
\text { großer zusätzlicher Aufwand). }\end{array}$ \\
\hline Rückblick & $\begin{array}{l}\text { Geschehnisse der } \\
\text { Anfangsphase } \\
\text { (bevor die beglei- } \\
\text { tende Forschung } \\
\text { einsetzen konnte) } \\
\text { ex post festhal- } \\
\text { ten. }\end{array}$ & $\begin{array}{l}\text { Reflexion des Anfangs- } \\
\text { prozesses anregen, } \\
\text { Gruppenidentität er- } \\
\text { höhen. }\end{array}$ & $\begin{array}{l}\text { Interner Blick auf den Prozess } \\
\text { möglich. Ansonsten für die } \\
\text { Beforschung Verlorenes zu- } \\
\text { mindest rekonstruieren. } \\
\text { Einzelne Aspekte geraten } \\
\text { schnell in Vergessenheit. }\end{array}$ \\
\hline
\end{tabular}

\section{Betreuung (organisatorisch)}

Eine gute Organisation ist ein wesentlicher Baustein für ein gelingendes Realexperiment. Über die neunmonatige Experimentlaufzeit werden die Experimentgruppen deshalb kontinuierlich von den Wissenschaftler(inne)n betreut.

Eine zentrale Methode stellen dabei Treffen mit den Mitgliedern aller Experimentgruppen dar. Diese Gruppentreffen bezwecken die Vernetzung und den Austausch zwischen den verschiedenen Experimentgruppen. Ein jeweils kurzer Statusbericht klärt über den aktuellen Stand der verschiedenen Realexperimente auf. Die anschließend durch die Wissenschaftler(innen) moderierten Gespräche dienen der Diskussion, Inspiration und Hilfestellung zwischen den Experimentie- 
renden. Die Gruppentreffen sollten in einem offenen Rahmen gesellig, freundlich und nicht zu formell gestaltet sein.

Genügend Zeit für Gespräche und Austausch ist hier entscheidend, Snacks und Getränke sind hilfreich für eine gute Atmosphäre. Die Gruppentreffen stellen einen intimen Rahmen, exklusiv für die Mitglieder der Experimentgruppen, dar. Die Treffen finden in regelmäßigem Abstand statt, werden protokollarisch festgehalten, und dienen den Experimentierenden als Grundgerüst und Orientierungspunkt, in denen auch über den Stand der Beforschung (Interviews, Fragebögen, Fokusgruppe) informiert wird.

Um einen guten Kontakt zu den Experimentierenden zu pflegen, empfiehlt es sich, direkte Ansprechpartner(innen) zu etablieren. Von jeder Experimentgruppe wird eine Person für die Kommunikation mit den Wissenschaftler(inne)n bestimmt. Diese Person ist auch für das Weiterleiten von Informationen innerhalb der Experimentgruppe verantwortlich. Seitens der Wissenschaftler(innen) sollte es ebenfalls ein bis maximal zwei Ansprechpersonen geben. Für alle Aktivitäten/Veranstaltungen, aber auch für die laufende Kommunikation benötigt es viel Organisations-, Abstimmungs- und Vorbereitungsarbeit durch die Wissenschaftler(innen). Daher ist es grundlegend, für die Kommunikation ausreichend Kapazitäten einzuplanen.

Während der Durchführung der gesamten Realexperimentreihe (wie auch bereits in der Phase ihrer Vorbereitung) erleichtert ein wöchentlich stattfindender Termin den kontinuierlichen Austausch und die Aufgabenverteilung unter den beteiligten Wissenschaftler(inne)n.

\section{Begleitung der Experimentierenden (inhaltlich)}

Neben den rein organisatorischen Aufgaben spielen der persönliche Kontakt mit den Experimentgruppen und die Art der Kommunikation auch aus anderen Gründen eine wichtige Rolle. Gerade zu Beginn eines Experiments bestehen oft Unsicherheit und Skepsis seitens der Experimentierenden, die mit Prozessen und Abläufen wissenschaftlicher Arbeit nicht vertraut sind. Es kann die Angst aufkommen, als Versuchskaninchen missbraucht zu werden. Vor Experimentstart sollten in einem oder mehreren Gesprächen die inhaltliche Ausrichtung der Realexperimente geklärt und die Forschungsinteressen seitens der Wissenschaftler(innen) transparent gemacht werden. Auch während der Experimentlaufzeit sollten diese für inhaltliche Fragen und Hilfestellungen zur Verfügung stehen.

Eine ehrliche und offene Kommunikation auf Augenhöhe bildet die Grundlage für eine gute, vertrauensvolle Zusammenarbeit. Der Aufbau eines Vertrauensverhältnisses zu den Experimentgruppen spielt eine tragende Rolle. Es ist wichtig, den Experimentierenden Wertschätzung für die von ihnen (zumeist ehrenamtlich) geleistete Arbeit entgegenzubringen und mit Geduld und Offenheit auf Feedback 
und Fragen zu reagieren. Durch den Einbau von Rückkopplungsschleifen im Forschungsdesign (etwa durch Feedbackmöglichkeiten bei den Gruppentreffen, durch Kritikmöglichkeiten am Ende jedes Fragebogens oder durch die Möglichkeit, jederzeit ins direkte Gespräch mit den Wissenschaftler(inne)n kommen zu können) fühlen sich die Experimentgruppen und die einzelnen Experimentierenden in ihren Anliegen zusätzlich ernst genommen. Ein kontinuierlicher beidseitiger Informationsaustausch erzeugt Transparenz und baut evtl. Ängste weiter ab. Darüber hinaus können auch die Gruppentreffen genutzt werden, um über geplante nächste Schritte zu informieren und einen offenen Raum für Fragen zu bieten. Die Wissenschaftler(innen) sollten von Anfang an vermitteln, dass es sich um ein ergebnisoffenes Experiment handelt, dass viel ausprobiert und auch mal etwas misslingen darf. Der Weg ist aus Forschungssicht ebenso interessant wie das Ziel. Dies klarzustellen, ist wichtig, um durch die Experimentierenden einen ehrlichen und ungeschönten Einblick in die Prozesse zu erhalten. Einen sicheren Raum für einen bewertungsfreien Austausch zu etablieren, ist eine zentrale Aufgabe der Begleitung der Realexperimente durch die Wissenschaftler(innen). Bei einer guten Gruppendynamik können die Gruppentreffen hierzu einen großen Beitrag leisten. Begleitung bedeutet auch, im Konfliktfall Hilfestellung in Form von Mediation anzubieten (oder diese selbst in Anspruch zu nehmen, falls Wissenschaftler(innen) in den Konflikt involviert sind). Diese Hilfestellung bezieht sich sowohl auf inhaltliche als auch auf persönliche Konflikte. Hier gilt es, immer die Diskussion und den Austausch zu suchen, eine Richtungsentscheidung, von oben sollte nur als allerletzte Möglichkeit in Betracht gezogen werden. Eventuell kann eine professionell geführte Mediation hilfreich sein (für weiterführende Literatur und Hilfestellung zum Thema Mediation s. a. BMLFUW 2016; Besemer 2009).

\section{Begleitung der Wissenschaftler(innen) (Supervision)}

Die in die Betreuung und Begleitung eines Realexperiments involvierten Wissenschaftler(innen) sollten während der Experimentlaufzeit von externen, nicht in der Realexperimentreihe tätigen Kolleg(inn)en durch Supervision begleitet werden. Insbesondere, um deren Rollenvielfalt zu reflektieren, um ggf. immanente/persönliche Rollenkonflikte aufzudecken und zu bearbeiten, und um immer wieder Distanz zum eigenen Tun und Forschen herzustellen. Eine solche Supervision eröffnet auch immer wieder neue Blickwinkel auf die eigene Rolle im Realexperiment und letztlich auch auf die Durchführung und Beforschung der Realexperimente. Sie gibt den Wissenschaftler(inne)n des Weiteren die Möglichkeit, schwierige Situationen (im Experimentverlauf, im Umgang mit den Experimentierenden etc.) sowie Zweifel am eigenen Tun anzusprechen. Die Begleitung der Wissenschaftler(innen) findet optimalerweise in einem kontinuierlichen Rhythmus statt. Diese Supervision ist für die Begleitung der Realexperimente sehr hilfreich (im Grunde ist sie unabdingbar). 


\section{Reflexion}

Insgesamt kann die Realexperimentreihe DNE, die im Reallabor Karlsruhe durchgeführt wurde, als erfolgreich bezeichnet werden. Die Resonanz auf den Wettbewerb, die Beteiligung, die Motivation und das Engagement der Experimentgruppen lag weit über den Erwartungen. Im Zuge von DNE wurden neue Transformationsprozesse für eine Nachhaltige Entwicklung in Karlsruhe angestoßen und soweit sich das bis dato sagen lässt - auch verstetigt. Waren die Experimente auf einen Zeitraum von neun Monaten begrenzt, so sind nach deren offiziellen Ende die Mehrheit der Mitglieder der Experimentgruppen auch ohne Unterstützung durch das Reallabor noch aktiv. Strukturen haben sich gefestigt und dadurch sind die Gruppen teils zu etablierten Akteuren im städtischen (Sozial-)Gefüge geworden.

Im Folgenden wird zuerst konkret auf die Resonanz zu den in DNE eingesetzten Methoden eingegangen. Anschließend werden Handlungsempfehlungen ausgesprochen, die für die Durchführung einer Realexperimentreihe generell bedeutsam sind, bevor abschließend das Potential dieses Formats als Raum zukünftiger Lern- und Bildungsprozesse aufgezeigt wird.

\subsection{Zur Resonanz der eingesetzten Methoden in „Dein NachhaltigkeitsExperiment“"}

Der Rücklauf bei den verschiedenen schriftlichen Befragungen und bei der Selbstdokumentation war im Vergleich zu klassischen sozialwissenschaftlichen Erhebungen extrem hoch (so belief sich der Rücklauf der 72 ausgeteilten Fragebögen auf 58 Stück, auf $80 \%$ ). Dies lässt sich wohl vor allem mit der starken Nähe des Reallabor-Teams zu den Experimentgruppen und der hohen Motivation der Experimentierenden erklären.

Allerdings war der Umgang mit den verschiedenen Forschungsmethoden und der Rücklauf zwischen den einzelnen Experimentgruppen unterschiedlich, und im Detail traten auch Schwierigkeiten auf. So taten sich die kleineren Experimentgruppen (3 Experimentierende) mit der Forderung der Protokollführung schwer, da viele Entscheidungen nicht in offiziellen Sitzungen getroffen wurden, während dies in den größeren Experimentgruppen (6-15 Experimentierende) mit regelmäBigen und offiziellen Treffen reibungslos verlief. Auch die Veranstaltungsdokumentation erwies sich in der für alle Experimente standardisierten Form nicht für alle Experimentgruppen als handhabbar und wurde für einzelne Experimente nochmals modifiziert. Die dreiteilige Fragebogenserie löste, wie sich im Zuge des zweiten Gruppentreffens zeigte, bei einigen Experimentierenden Stress aus. Die Angst, etwas falsch zu machen, wurde als große Hürde beim Ausfüllen angegeben und führte teilweise dazu, dass Fragebögen nicht abgegeben wurden. Zudem 
wurden einzelne Fragen oder Fragengruppen als zu komplex wahrgenommen, etwa jene, die sich mit dem persönlichen Nachhaltigkeitsverständnis befassten. Rückblickend kann gesagt werden, dass vielen Teilnehmer(inne)n geführte mündliche Befragungen wie Gruppeninterviews oder die Fokusgruppe leichter fielen als schriftliche Befragungen.

Die Gruppentreffen schließlich, bei denen sich alle an DNE beteiligten Experimentgruppen trafen, wurden gut angenommen, trugen zur Klärung bei, inspirierten und wurden zum Austausch genutzt. Hier entwickelte sich bereits nach dem ersten Treffen eine Dynamik der gegenseitigen Unterstützung und des sehr offenen, gegenseitigen Austauschs.

Die eingesetzten Moderationsmethoden leisteten gute Dienste, wurden von den Beteiligten kaum als solche wahrgenommen - zumindest nicht als fremd oder störend - und trugen sehr zur Gestaltung, Atmosphäre und Produktivität der Gruppentreffen, Interviews, Fokusgruppe und der weiteren Aktivitäten/Veranstaltungen bei. Dass zwei Mitglieder des Reallabor-Teams über eine Moderationsausbildung verfügten, erwies sich als überaus hilfreich.

Die intensive, aufeinander aufbauende und auch graphisch aufwändig aufbereitete Kommunikationsstrategie (Kurzfilme, Postkarten, Poster, Blog-Artikel, Facebook und Twitter) führte zu einer hohen Zahl an Einsendungen für den Wettbewerb und einer großen Bandbreite an vorgeschlagenen Ideen. Allerdings konnten nicht alle zivilgesellschaftlichen Gruppen erreicht werden. So fanden sich keine Bürger(innen) mit Migrationshintergrund unter den Bewerber(inne)n für den Wettbewerb, und auch in den mit der Zeit wachsenden Experimentgruppen waren diese wenig involviert.

\subsection{Empfehlungen zur Durchführung einer Realexperimentreihe}

Eine Realexperimentreihe wie DNE durchzuführen, gelingt nur in einem Nahverhältnis zwischen den Wissenschaftler(inne)n und den Experimentierenden. Dies kann eine fruchtbare Bildungssituation schaffen, sofern die Wissenschaftler(innen) und die Experimentierenden sich offen, transparent und auf Augenhöhe begegnen. Ein partnerschaftlicher, zugewandter und authentischer Umgang stellt die Basis für eine gute Zusammenarbeit dar und trägt entscheidend zum Gelingen bei. Ein solcher Umgang erfordert von den Wissenschaftler(inne)n die Bereitschaft, vom Forschungs- und Selbstbild eines/einer neutralen, außenstehenden, quasi-objektiven Forschenden Abstand zu nehmen und sich mit in das Realexperiment zu begeben. Die nötige Distanz zum Forschungsgegenstand muss immer wieder über Selbst- und Gruppen-Reflexionen und bestenfalls Supervision durch nicht am Realexperiment beteiligte Kolleg(inn)en erarbeitet werden. 
Hinzu kommt, dass Wissenschaftler(innen) in solchen Vorhaben neben der Forschungstätigkeit mit einer besonders hohen Anzahl und Intensität weiterer Tätigkeiten wie Projektorganisation, Kommunikation oder Mediation konfrontiert werden, die in der Projektplanung oftmals unterschätzt werden. Teile solcher Tätigkeiten können auch durch eine erfahrene wissenschaftsnahe Kommunikationsund Moderationsagentur übernommen werden, um die Wissenschaftler(innen) zu unterstützen. Dabei ist aber zu beachten, dass die enge inhaltliche Anbindung an die Wissenschaft gewährleistet sein muss.

Das Gelingen bzw. Misslingen eines Realexperiments umfasst gemäß seinen divergierenden Zielsetzungen mehrere Dimensionen. So kann es in seiner transformativen Dimension scheitern, also z. B. keine Beiträge für eine Nachhaltige Entwicklung erzeugen, aber dennoch wissenschaftlich wertvolle Erkenntnisse etwa zu Erfolgs- und Misserfolgsfaktoren liefern. Oder es kann in der transformativen Dimension gelingen, jedoch kaum wissenschaftliche Ergebnisse liefern aber in seiner Bildungsdimension wichtige Lernprozesse anstoßen. Die voneinander unabhängigen Gelingensdimensionen von Realexperimenten gilt es insofern zunächst getrennt zu erfassen, und in der Zusammenarbeit mit den Kooperationspartnern sollten die unterschiedlichen Zielsetzungen zu Beginn herausgearbeitet und transparent gemacht werden. Ein weiterer wichtiger Faktor bei einer Realexperimentreihe wie DNE ist die Zeit. Dies umfasst zum einen die für die Vertrauensbildung benötigte Zeit für Kommunikation, physische Treffen - auch im informellen Rahmen - und gemeinsame Tätigkeiten.

Es umfasst aber auch die Konzeption des Forschungsprogramms, die durch eine solche Anlage erst parallel zu den bereits startenden Experimenten erfolgen kann, weil sowohl die Realexperimente als auch die Zusammensetzung der Experimentgruppen erst mit Ende des Wettbewerbs feststehen, beides aber Implikationen für die Beforschung hat. Hier muss dementsprechend zu einem bestimmten Zeitpunkt schnell und präzise über das Forschungsdesign entschieden und dies rasch umgesetzt werden. Aufgrund der knappen Zeit gilt es dabei sehr genau zu überlegen, welche Daten zur Auswertung tatsächlich benötigt und im geplanten Zeitraum ausgewertet werden können. Selbstverständlich sollte auch für die Reflexion, Auswertung und Veröffentlichungen der Forschungen nach Abschluss der Experimentreihe ausreichend Zeit eingeplant werden.

Für die enge Zusammenarbeit mit Bürger(inne)n müssen die involvierten Wissenschaftler(innen) neben ihrem Fachwissen auch in erhöhtem Maße soziale Kompetenzen mitbringen. Auch eine Sensibilität dafür, was den Experimentierenden an Forschungsaktivitäten zumutbar ist, sollte im gesamten Projektverlauf präsent sein. Hier sind Verständnis und Geduld mitzubringen, bei Problemen oder Verzögerungen ist behutsam nachzufragen und ggf. sind Anpassungen am Forschungsdesign vorzunehmen. 
Auch muss das Team der Wissenschaftler(innen) bei Konflikten innerhalb der Realexperimente abwägen, inwieweit es eingreifen soll. Hier gilt ebenfalls: Ist eine Vertrauensbasis vorhanden, so ist eine gelingende Intervention deutlich aussichtsreicher. Letztlich sollten die Experimente auch Gegenstand ethischer Reflexion werden. Als grobe Orientierung empfiehlt sich, im (moralischen, realweltlichen) Zweifelsfall, die wissenschaftlichen Interessen hinter das Wohl der Beteiligten zurückzustellen.

Abschließend empfehlen wir einen realen Ort, der nutzbare Infrastruktur, Anlaufstelle bei Fragen und Austauschplattform für die Experimentgruppen ist, zu etablieren. Dadurch wird das Reallabor greifbar, ansprechbar und Bürger(innen) können sich leichter mit diesem identifizieren. In der Realexperimentreihe DNE hat sich der ,Zukunftsraum für Nachhaltigkeit und Wissenschaft' als solcher Ort sehr bewährt, wie es auch aus den Erhebungen bei den Experimentierenden hervorgeht.

\subsection{Ausblick: Realexperimente als Bildungsanlass}

Die Erfahrung mit DNE hat gezeigt, dass sich mit der Durchführung von Realexperimenten fruchtbare Bildungsprozesse auf ganz unterschiedlichen Ebenen entfalten können, auf der individuellen wie auf der gemeinschaftlichen Ebene. Bildungsprozesse haben bei DNE sowohl im Bereich der Wissensvermittlung und -erarbeitung stattgefunden, als auch insbesondere im Aufbau von persönlichen Kompetenzen, Selbstreflexion und Selbstbildung. Zudem wurde gemeinschaftliches Empowerment angestoßen und gelebt. So wurde im Falle von DNE der Wissenshorizont der Experimentierenden erweitert, indem diese zum Beispiel zu Konferenzen reisten, Fachvertreter(innen) zum Austausch einluden und selbst Vorträge zu ihren spezifischen Experimentthemen (z. B. artgerechte Bienenhaltung) gaben. Darüber hinaus kamen viele durch die Themenschwerpunkte der Realexperimente zum ersten Mal direkt in Kontakt mit abstrakt scheinenden Ansätzen wie Entschleunigung. Hier wurden durch das Herstellen realweltlicher Bezüge und den Erfahrungsaustausch Denkprozesse angestoßen, die langfristig ihre Wirkung entfalten können. Der begleitenden Forschung kam so auch eine didaktische Rolle zu.

Es kann also angenommen werden, dass Realexperimente $\mathrm{zu}$ formellem und informellem Lernen beitragen (s. generell zu Bildungszielen in Reallaboren Beecroft et al. 2018). Aber auch für die beteiligten Wissenschaftler(innen) vollzieht sich, neben der Erkenntnisproduktion, ein persönlicher Bildungsprozess. Bei der Durchführung einer Realexperimentreihe wird daher abschließend ein Fokus auf die Bildungsaspekte in der Konzeption, in der Gestaltung sowie bei der wissenschaftlichen Erfassung empfohlen. 


\section{Dank}

Die Autorinnen und der Autor danken Monika Bachinger, Thomas Becker, Raphael Dietz, Maximilian Haag, Barbara Koch, Jan A. Lutz, Editha Marquardt, Hanna Noller, Rasmus Prieß und Eric Puttrowait für die Diskussionen über frühere Fassungen des Textes. Insbesondere möchten sie Sophia Alcántara und Regina Rhodius sowie zwei anonymen Gutachter(inne)n für die konkreten Hinweise zur Verbesserung des Textes im Rahmen des internen und externen Reviews danken. Schließlich danken die Autorinnen und der Autor den beiden Herausgebenden, Rico Defila und Antonietta Di Giulio, für ihre Rückmeldungen zum Text.

\section{Literatur}

Albiez, M., Meyer-Soylu, S., Parodi, O., \& Waitz, C. (2016). Das „Quartier Zukunft Labor Stadt“: ein reales Reallabor. In: U. Hahne \& H. Kegler (Hrsg.): Resilienz. Stadt und Region - Reallabore der resilienzorientierten Transformation (S. 101-125). Peter Lang.

Arbter, K. (2012): Praxisbuch Partizipation. Gemeinsam die Stadt entwickeln. Werkstattbericht N. 127, Magistrat der Stadt Wien.

Arnold, A., \& Piontek, F. M. (2018). Zentrale Begriffe im Kontext der Reallaborforschung. In R. Defila \& A. Di Giulio (Hrsg.), Transdisziplinär und transformativ forschen. Eine Methodensammlung (S. 143-154). Wiesbaden: Springer VS.

Beecroft, R., \& Parodi, O. (2016). Einführung in den Schwerpunkt. Technikfolgenabschätzung - Theorie und Praxis, 25 (3), (S. 4-8). Online verfügbar unter http:// www.tatup-journal.de/downloads/2016/tatup163.pdf.

Beecroft, R., Trenks, H., Rhodius, R., Benighaus, C., \& Parodi, O. (2018). Reallabore als Rahmen transformativer und transdisziplinärer Forschung: Ziele und Designprinzipien. In R. Defila \& A. Di Giulio (Hrsg.), Transdisziplinär und transformativ forschen. Eine Methodensammlung (S. 75-100). Wiesbaden: Springer VS.

Besemer, C. (2009). Mediation. Die Kunst der Vermittlung in Konflikten, Werkstatt für gewaltfreie Aktion Baden.

BMLFUW (2016): Mediation. Partizipation \& nachhaltige Entwicklung in Europa, Informationswebsite des Bundesministeriums für Land- und Forstwirtschaft, Umwelt und Wasserwirtschaft. http://www.partizipation.at/mediation.html. Zugegriffen am 20.12.2017.

Defila, R., \& Di Giulio, A. (2018). Reallabore als Quelle für die Methodik transdisziplinären und transformativen Forschens - eine Einführung. In R. Defila \& A. Di Giulio (Hrsg.), Transdisziplinär und transformativ forschen. Eine Methodensammlung (S. 935). Wiesbaden: Springer VS.

Di Giulio, A., Defila, R., \& Brückmann, Th. (2016). „Das ist halt das eine ... Praxis, das andere ist Theorie" - Prinzipien transdisziplinärer Zusammenarbeit im Forschungsalltag. In: R. Defila \& A. Di Giulio (Hrsg.): Transdisziplinär forschen - zwischen 
Ideal und gelebter Praxis. Hotspots, Geschichten, Wirkungen (S. 189-286). Frankfurt a. M., New York: Campus.

Hammer, A., Ott, I., \& Stiller, S. (2014). Karlsruhe Oststadt: Heute und in Zukunft. Karlsruhe.

Helfferich, C. (2014). Leitfaden- und Experteninterview. In: N. Baur \& J. Blasius (Hrsg.): Handbuch Methoden der empirischen Sozialforschung. Wiesbaden: Springer VS.

Henseling, C., Hahn, T., \& Nolting, K. (2006). Die Fokusgruppen-Methode als Instrument in der Umwelt- und Nachhaltigkeitsforschung. Institut für Zukunftsstudien und Technologiebewertung. Berlin: WerkstattBericht Nr. 82.

Kopfmüller, J., Brandl, V., Jörissen, J., Paetau, M., Basne, G., Coenen, R., \& Grunwald, A. (2001). Nachhaltige Entwicklung integrativ betrachtet. Konstitutive Elemente, Regeln, Indikatoren. Berlin: editionsigma.

Mayer, H. O. (2013). Interview und schriftliche Befragung. Grundlagen und Methoden empirischer Sozialforschung. 6. überarbeitete Aufl. München: Oldenbourg Wissenschaftsverlag.

Meyer-Soylu, S., Parodi, O., Trenks, H., \& Seebacher, A. (2016). Das Reallabor als Partizipationskontinuum. Erfahrungen aus dem Quartier Zukunft und Reallabor 131 in Karlsruhe. Technikfolgenabschätzung - Theorie und Praxis, 25 (3), (S. 31-40). Online verfügbar unter http://www.tatup-journal.de/downloads/2016/tatup163.pdf.

Parodi, O., Albiez, M., Beecroft, R., Meyer-Soylu, S., Quint, A., Seebacher, A., Trenks, H., \& Waitz, C. (2016). Das Konzept „Reallabor“ schärfen. Ein Zwischenruf des Reallabor 131: KIT findet Stadt. GAIA 25 (4), (S. 284-285). doi: 10.14512/gaia.25.4.11.

Parodi, O., Beecroft, R., Albiez, M., Quint, A., Seebacher, A., Tamm, K., \& Waitz, C. (2016). Von „Aktionsforschung“ bis „Zielkonflikte“. Schlüsselbegriffe der Reallaborforschung. Technikfolgenabschätzung - Theorie und Praxis, 25 (3), (S. 9-18). Online verfügbar unter http://www.tatup journal.de/downloads/2016/tatup163.pdf.

Porst, R. (2014). Fragebogen. Ein Arbeitsbuch. 4. Aufl. Wiesbaden: Springer VS.

Rosa, H. (2014). Beschleunigung. Die Veränderung der Zeitstrukturen in der Moderne. 10. Aufl. Frankfurt a. M.: Suhrkamp Taschenbuch.

Seebacher, A., Alcántara, S., \& Quint, A. (2018). Akteure in Reallaboren - Reallabore als Akteure. In R. Defila \& A. Di Giulio (Hrsg.), Transdisziplinär und transformativ forschen. Eine Methodensammlung (S. 155-159). Wiesbaden: Springer VS.

Sommer, J. (Hrsg.). (2015). Kursbuch Bürgerbeteiligung. Berlin: Verlag der deutschen Umweltstiftung.

Waitz, C., \& Meyer-Soylu, S. (2016). Das ReparaturCafé als Transformationselement im urbanen Raum. Technikfolgenabschätzung - Theorie und Praxis, 25 (2), S. 22-28. Online verfügbar unter http://www.tatup-journal.de/tatup162.php.

Werkstatt für Gewaltfreie Aktion Baden (Hrsg.). (2004). Konsens. Handbuch zur gewaltfreien Entscheidungsfindung. 1. Aufl. Karlsruhe: Eigenverlag. 


\section{Anhang}

Anlage a: Aufruf zum Wettbewerb „Dein NachhaltigkeitsExperiment“ im Mai 2016 (Poster)

\section{»Dein NachhaltigkeitsExperiment«}

\#ZukunftAnzetteln

\section{Gemeinschaft \& Entschleunigung anstiften!}

Was geschieht wenn du dein Leben änderst? Im Sommer 2016 startet »Dein NachhaltigkeitsExperiment « in der Karlsruher Oststadt.

Bis zu 12000 Euro für Eure Experimente Du willst mitmachen und \#ZukunftAnzetteln? Mehr Infos gibt's unter www.quartierzukunft.de.

Bewerbungsschluss ist der 12. Juni, die Experimente sind auf 9 Monate angelegt (Juli bis März). Die Förderung wird vorraussichtlich auf 3 bis 4 Experimente verteilt. Mitmachen können Teams ab 3 Personen (gerne mehr). Weitere Infos und die genauen Teilnahmebedingungen auf unserer Website oder per QR Code.

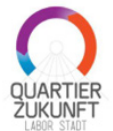

http://quartierzukunft.de/ dein-nachhaltigkeitsexperiment/

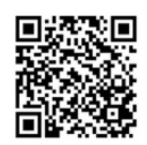


Anlage b: Teilnahmebedingungen und Bewerbungsverfahren für den Wettbewerb zu „Dein NachhaltigkeitsExperiment“

\section{Teilnahmebedingungen und Bewerbungsverfahren}

\section{zu „DeinNachhaltigkeitsExperiment"}

- Das Experiment soll die Themen Gemeinschaft und/oder Entschleunigung zum Inhalt haben. Es ist wünschenswert beide Themen zu verbinden und noch weitere Nachhaltigkeitsaspekte zu berücksichtigen (vgl. z.B. Leporello).

- Das Experiment hat eine Laufzeit von neun Monaten (Anfang Juli 2016 bis Ende März 2017).

— Es werden voraussichtlich drei bis vier Experimente gefördert.

- Das Quartier Zukunft und die Bürgerstiftung Karlsruhe fördern die ausgewählten Experimente mit insgesamt bis zu $\mathbf{1 2 . 0 0 0} €$. Das Geld wird unter den Gewinnern nach Ermessen der Jury aufgeteilt. Es ist ausschließlich zur Durchführung des Experiments vorgesehen. Die Auszahlung erfolgt auf zwei Raten. Bei einem vorzeitigen Abbruch des Experiments behält sich die Jury vor, nicht die gesamte Förderung auszuzahlen.

— Teilnahmeberechtigt sind alle natürlichen Personen ab 18 Jahren, gemeinnützige Organisationen und zivilgesellschaftliche Gruppen.

- Das Experiment soll in der Karlsruher Oststadt verortet sein oder zumindest starken Oststadtbezug aufweisen.

- Das Experiment darf noch nicht begonnen haben, kann aber in ein bestehendes Projekt eingebettet sein.

- Das Kernteam des Experiments muss aus mindestens drei Personen bestehen, die bei Wettbewerbsteilnahme feststehen. Es sollen aber im Verlauf gerne mehr Menschen involviert werden. Die BewerberInnen sollen aufrichtig bereit sein, Gemeinschaft und Entschleunigung in Ihr eigenes Leben einzubeziehen.

- Die BewerberInnen erklären sich bereit, an der wissenschaftlichen Begleitung mitzuwirken. Insbesondere:

- für Interviews und Fragebögen zur Verfügung zu stehen

- eine eigenständige visuelle oder textliche Dokumentation zu erstellen, z.B. Fotooder Videodokumentation, Tagebuch

- an den Gruppentreffen (Einführungsveranstaltung, Zwischen- und Abschlusstreffen) teilzunehmen 
festgelegt.

- Bewerbungszeitraum ist der 02. Mai bis 12. Juni. Bewerbungen können per E-Mail oder Post eingereicht werden. Für eine postalische Einreichung gilt der Poststempel vom 10. Juni.

- Durch eine Vorauswahl bestimmte BewerberInnen, können sich und ihre Experimentideen der Jury am Donnerstag, den 16. Juni zwischen 18.30 und 20.30 Uhr vorstellen.

- Die Entscheidung über die Preisvergabe wird am 22. Juni auf der Quartier ZukunftWebsite bekanntgegeben. Die Gewinner werden zudem per E-Mail verständigt.

— Eine Bewerbung beinhaltet:

- Bewerbungsformular mit vorläufigem Zeit- und Budgetplan

- Download Bewerbungsformular

- Das Bewerbungsformular kann auch im Zukunftsraum abgeholt werden

- formlose Vorstellung der Experimentidee (Poster, Film, Text o.ä.)

\begin{tabular}{|c|c|c|}
\hline \multicolumn{3}{|c|}{ MÖGLICHE KONTAKTADRESSEN } \\
\hline E-Mail & Adresse Institut & Zukunftsraum \\
\hline helena.trenks@kit.edu & $\begin{array}{l}\text { KIT } \\
\text { ITAS } \\
\text { Helena Trenks } \\
\text { Karlstr. } 11 \\
76133 \text { Karlsruhe }\end{array}$ & $\begin{array}{l}\text { Zukunftsraum für Nachhaltigkeit und } \\
\text { Wissenschaft } \\
\text { Helena Trenks } \\
\text { Rintheimer Str. } 46 \\
76131 \text { Karlsruhe }\end{array}$ \\
\hline
\end{tabular}

Die Teilnahme erfolgt freiwillig. Es besteht kein Rechtsanspruch auf die Auszahlung der Mittel. 
Anlage c: Veranstaltungsdokumentation (Vorlage zuhanden der Experimentgruppen)

\title{
VERANSTALTUNGSDOKUMENTATION
}

\author{
NachhaltigkeitsExperiment:
}

$\cdots$

Name und Art der Veranstaltung:

Datum\& Zeitraum der Veranstaltung:

Wo fand die Veranstaltung statt?

$\cdots$

Was war das Ziel der Veranstaltung?

$\cdots$

Ist das Ziel der Veranstaltung erreicht worden?Wenn ja, inwiefern? Wenn nicht, was können die Gründe dafür sein?

Wie viele BesucherInnen waren im gesamten Zeitraum ungefähr anwesend? Wie war die Altersstruktur?

Wie war die Resonanz der BesucherInnen auf die Veranstaltung?Wie war die Stimmung? (eigene Einschätzung, gerne auch mündliche Zitate wiedergeben)

Nahmen die BesucherInnen der Veranstaltung persönlich etwas mit nach Hause? Wenn ja, was? 
Welche Nachhaltigkeitsthemen wurden auf der Veranstaltung thematisiert?

(detailliertere Informationen im Leporello „Wie Nachhaltigkeit möglich ist")

\begin{tabular}{|l|l|}
\hline IKoNE Regel [s. Kopfmüller et al. 2001] & thematisiert (x) \\
\hline 1.1 Schutz der menschlichen Gesundheit & \\
\hline 1.2 Gewährleistung der Grundversorgung & \\
\hline 1.3 Selbstständige Existenzsicherung & \\
\hline 1.4 Gerechte Verteilung der Umweltnutzungsmöglichkeiten & \\
\hline 1.5 Ausgleich extremer Einkommens- und Vermögenunterschiede & \\
\hline 2.1 Nachhaltige Nutzung erneuerbarer Ressourcen & \\
\hline 2.2 Nachhaltige Nutzung nicht-erneuerbarer Ressourcen & \\
\hline 2.3 Nachhaltige Nutzung der Umwelt als Senke & \\
\hline 2.4 Vermeidung unvertretbarer technischer Risiken & \\
\hline 2.5 Nachhaltige Entwicklung des Sach-, Human- und Wissenskapitals & \\
\hline 3.1 Chancengleichheit im Hinblick auf Bildung, Beruf, Information & \\
\hline 3.2 Partizipation an gesellschaftlichen Entscheidungsprozessen & \\
\hline 3.3 Erhaltung des kulturellen Erbes und der kulturellen Vielfalt & \\
\hline 3.4 Erhaltung der kulturellen Funktion der Natur & \\
\hline 3.5 Erhaltung der sozialen Ressourcen & \\
\hline
\end{tabular}

\section{Weitere adressierte Themen?}

...

Wurden die Themen Gemeinschaft und Entschleunigung thematisiert? Wenn ja, wie? ...

Wirkte die Veranstaltung entschleunigend auf die BesucherInnen? Wenn ja, inwiefern? $\cdots$

Wirkte die Veranstaltung gemeinschaftsbildend auf die BesucherInnen?Haben sich diese wohlgefühlt? Wurden neue Kontakte geknüpft?

$\cdots$

\section{Sonstige Anmerkungen:}

$\ldots$ 
Open Access Dieses Kapitel wird unter der Creative Commons Namensnennung 4.0 International Lizenz (http://creativecommons.org/licenses/by/4.0/deed.de) veröffentlicht, welche die Nutzung, Vervielfältigung, Bearbeitung, Verbreitung und Wiedergabe in jeglichem Medium und Format erlaubt, sofern Sie den/die ursprünglichen Autor(en) und die Quelle ordnungsgemäß nennen, einen Link zur Creative Commons Lizenz beifügen und angeben, ob Änderungen vorgenommen wurden.

Die in diesem Kapitel enthaltenen Bilder und sonstiges Drittmaterial unterliegen ebenfalls der genannten Creative Commons Lizenz, sofern sich aus der Abbildungslegende nichts anderes ergibt. Sofern das betreffende Material nicht unter der genannten Creative Commons Lizenz steht und die betreffende Handlung nicht nach gesetzlichen Vorschriften erlaubt ist, ist für die oben aufgeführten Weiterverwendungen des Materials die Einwilligung des jeweiligen Rechteinhabers einzuholen. 